\title{
MULTI-PHASE MODELLING OF INTERGRANGULAR HYDROGEN SEGREGATION/TRAPPING FOR HYDROGEN EMBRITTLEMENT
}

\author{
${ }^{*}$ S.Jothi ${ }^{1}$, T.N. Croft ${ }^{1}$, L. Wright ${ }^{2}$, A. Turnbull ${ }^{2}$ and S.G.R.Brown ${ }^{1}$ \\ ${ }^{1}$ College of Engineering, Swansea University, Singleton Park, Swansea, SA2 8PP, UK. \\ ${ }^{2}$ National Physical Laboratory, Teddington, Middlesex TW11 0LW, UK. \\ *s.jothi@swansea.ac.uk
}

\begin{abstract}
Premature failure in polycrystalline materials due to hydrogen absorption affects a wide range of applications, including clean energy systems, hydrogen storage systems and rocket engines. A good understanding of the diffusion and trapping processes within such materials can inform material choices and component design to reduce the likelihood of such failures. Grain boundary segregation of hydrogen can often lead to intergranular hydrogen embrittlement (IHE). In order to understand the effects of hydrogen on intergranular and transgranular fracture in polycrystalline material it is important to first understand hydrogen diffusion and trapping in the general context of grain boundary segregation engineering (GBSE). Hydrogen diffusion is affected by local microstructural features including intergranular second phase precipitates, grain boundary (GB) thicknesses and geometrically necessary dislocation (GND) density. A multi-scale multi-phase model is presented here that has been developed to study GBSE with respect to hydrogen diffusion and IHE. The results of various multi-scale GBSE models with and without traps(including the effects of microstructure, intergranular precipitate phases and GB thickness) are compared and discussed, and the effects of microstructural parameters such as hydrogen segregation factor and GND trapping density on hydrogen diffusion are investigated.
\end{abstract}

Keywords: Hydrogen Embrittlement; Microstructures; Metallic Polycrystalline Material; User Element; Finite Element Analysis;

\subsection{INTRODUCTION}

Hydrogen is a common fuel in rocket engines and these rocket engines are made of polycrystalline materials. It is well known that hydrogen causes embrittlement in many polycrystalline materials, including high nickel content polycrystalline materials, and 
catastrophic failure can occur in hydrogen fuel rocket engine components [1-19]. Hydrogen induced intergranular and transgranular embrittlement in polycrystalline materials are well established as causes of catastrophic brittle failure and have been studied extensively for more than five decades [1-13]. Apart from rocket systems, hydrogen diffusion, segregation and trapping in metallic polycrystalline materials are also significant as causes of hydrogen embrittlement in clean energy fuel storage systems used as power sources in the aerospace and automotive sectors. Physical microstructural factors such as grain size, grain boundaries, dislocations, grain boundary segregation of impurities, carbides and non-metallic particles have been identified as playing important roles in this phenomenon [11, 20-50].

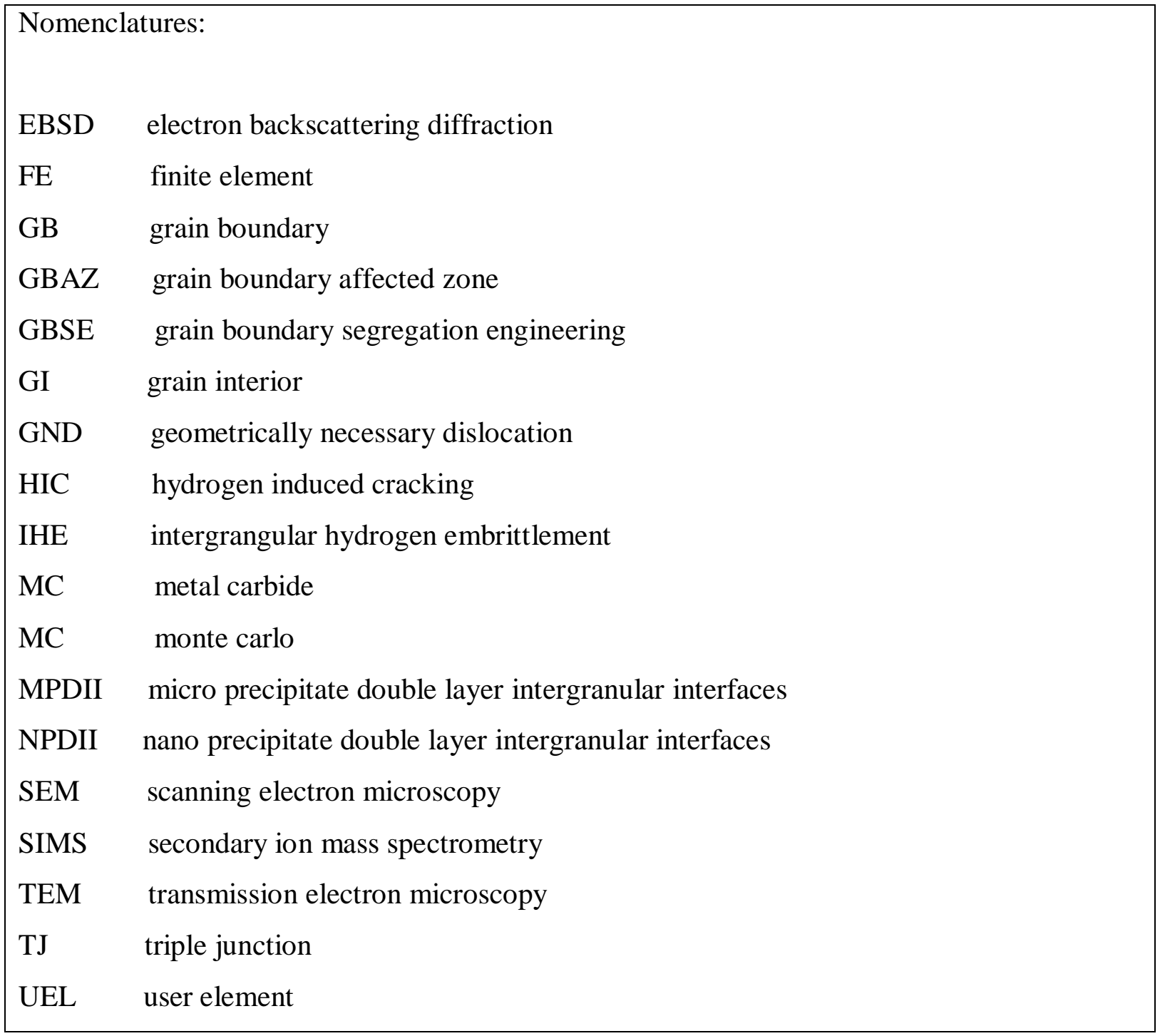

For example, segregation of phosphorous and sulphur assists hydrogen embrittlement in nickel based super alloys [46-47]. Some of these microstructural features such as grain boundaries (GBs) may also promote faster diffusion of hydrogen due to the locally disordered 
atomic structure [22-23, 32-41,44-45]. GBs may also act as hydrogen trap (or segregation) sites when the probability of atomic hydrogen jumping into GB sites (capture) is greater than that of atomic hydrogen jumping out of GB sites (escape) [51-54]. Grain boundary segregation engineering (GBSE) is fundamental to understanding intergranular hydrogen embrittlement [22,42,45,51,54]. Dislocation sites may also act as traps for hydrogen atoms (providing a deeper potential energy well) and hydrogen atoms jumping into these sites may reside for longer times than in lattice sites [51-54]. These trapping effects can lead to an overall decrease in the rate of hydrogen transport over time [11-12, 55-59]. The processes of hydrogen transport through/near such microstructural features are important for understanding the different modes of hydrogen induced cracking (HIC) in polycrystalline nickel [21-22] for example.

Intergranular and transgranular failures are two different modes of HIC. HIC commonly occurs along grain boundaries where high concentrations of hydrogen can exist [21]. These concentrations highlight the importance of including the random grain boundaries in any numerical model designed to predict embrittlement. Detailed description of the importance of grain boundary diffusion of hydrogen in nickel can be found in Harris [22, 51]. In nickel components combinations of micro polycrystalline and nanopolycrystalline nickel microstructures are often encountered. These are complex composites comprising irregular polygonal grains, random grain boundaries and triple junctions. The detailed nature of random irregularmicro and nano polygonal grains and random irregular grain boundaries is significant when considering the processes of hydrogen diffusion and segregation mechanisms that lead to embrittlement failure in nickel [22-24].

Manufacturing processes such as casting, electro deposition and welding of polycrystalline materials and post-manufacturing processes such as hot rolling, cold rolling, heat treatment and hardening using thermo-mechanical processing produces residual plastic strain and GB misorientation which is directly associated with (geometrically necessary dislocation) GNDs. In order to understand GBSE for hydrogen transport based on elastic and plastic strains developed by manufacturing processes, a trap model and multi-scale multiphase microstructural model has been developed to study the effect of GB misorientation, GND density and trap density on hydrogen segregation mechanisms in polycrystalline material. GB misorientations accommodate GNDs [32,45, 51, 60]. Hydrogen diffusion and segregation may increase or decrease depending on GB misorientation, GND density and GB energy, and GNDs can act as trapping sites for hydrogen in polycrystalline materials. It is also 
reported that hydrogen diffusion and segregation is influenced by GNDs, dislocation density, triple junctions (TJs), GB misorientation and GB connectivity [32,60-61]. The GND density may also vary depending on the GB misorientation types, angle and the GB energy. Previously hydrogen diffusion in FCC polycrystalline material has been studied using a Secondary ion Mass Spectrometry (SIMS) technique and it was reported that high energy GBs accelerate the diffusivity of hydrogen [25, 45, 54, 60, 62].

Raabe et al observed double nano layers in grain boundary interfaces with thicknesses of 10 to $15 \mathrm{~nm}$ in polycrystalline steel material. Impurity segregation and nanoprecipitation in this intergranular double layer was also reported [42-43]. Microprecipitation in intergranular double layers in nickel-based polycrystalline alloys have been observed in this work as shown in figure1. This indicates that it is important to take into account this intergranular double layer interface when studying the GBSE of impurity segregation and trapping in polycrystalline materials.

In the present study, several multi-scale multi-phase simulations with heterogeneous random irregular polygonal grains possessing random intergranular double layers as a second phase have been performed. Various microstructures containing intergranular double layer precipitate interfaces and Grain boundary affected zones (GBAZs) have been generated. A trap model based on the McNabb trap model has been implemented in a FORTRAN user element (UEL) subroutine within an ABAQUS-based multi-scale multi-phase microstructural model. Monte Carlo (MC) simulation has been performed for microstructural grain geometry development and trap density calculations based on GB misorientation. A coupled MC Finite Element (FE) multi-scale multi-phase continuum trap model is used to investigate hydrogen trapping and GB segregation mechanisms in polycrystalline material. A MC model has also been developed to investigate the microstructural effects of hydrogen segregation factor, GND trapping density and lattice hydrogen concentration on hydrogen diffusion in polycrystalline material. The behaviour of these synthetic microstructures using both non-trap and trap models is also reported.

\subsection{MATHEMATICAL MODELS FOR HYDROGEN TRANPORT WITH AND WITHOUT TRAP}

\subsection{Non-Trap hydrogen transport model:}


The non-trap transport of hydrogen is simply described by Fick's laws where solute diffuses from high concentration regions to low concentration regions based on the equations below

$\boldsymbol{J}_{\boldsymbol{c}}(\boldsymbol{X}, t)=-D \nabla C$

$\frac{\partial C}{\partial t}=\nabla \cdot(D \nabla C)$

Where $D$ is the effective diffusivity of hydrogen $\left(\mathrm{m}^{2} \mathrm{~s}^{-1}\right)$ and $C$ is hydrogen concentration. The hydrogen flux vector $J_{c}$ consists of hydrogen concentration gradient $\nabla C$ at a specific time $t$ based on Fick's first law of diffusion by using the thermodynamic formulation based on the Gibbs free enthalpy. Details of the development of the non-trap hydrogen diffusion microstructure model can be found elsewhere [9, 10, 33-35]. The material properties in the microstructural model include the within-grain hydrogen diffusion coefficient $D_{g}$ and the grain boundary hydrogen diffusion coefficient $D_{g b}$. The full finite element formulation of the non-trap model is described elsewhere [34-35, 63-65, 66].

\subsection{Trap hydrogen transport model:}

It has been reported previously that the GND density close to GBs is high when compared to the GND density in centre of the grains [67]. This high GND density region close to the GB is referred to as the grain boundary affected zone (GBAZ) throughout this paper. Higher GND densities are due to the geometrical misalignment of two adjacent grain orientations (i.e. GB misorientations). A numerical model must therefore be able to define different effective GBAZ thicknesses. So the non-trap models were used in grain region and trap models were used at grain boundary. The governing equation for hydrogen diffusion with trapping in the presence of grain boundary precipitate phases is:

$\frac{\partial C}{\partial t}=\frac{\partial C_{d i f f}}{\partial t}-\frac{\partial C_{t r a p}^{i}}{\partial t}$

The concentration flux is now determined by the normal grain boundary hydrogen diffusion and a concentration flux due to hydrogen trapping,

$\frac{\partial C_{\text {diff }}}{\partial t}=\nabla \cdot\left(D_{g b} \nabla C\right)$

$\frac{\partial C_{\text {trap }}^{i}}{\partial t}=N_{t}^{i} \frac{\partial n_{i}}{\partial t}$

$\frac{\partial n_{i}}{\partial t}=k_{i} C\left(1-n_{i}\right)-p_{i} n_{i}$

$N_{t}^{i=G N D}=\frac{\pi \cdot r^{2}}{2 d} \cdot N_{L} \cdot \sum_{j=1}^{m_{g b}} \frac{1}{S_{j}}$

$=\frac{\pi \cdot r^{2}}{b \cdot d} \cdot N_{L} \cdot \sum_{j=1}^{m_{g b}} \sin \left(\frac{\theta_{j}}{2}\right) \cdot f_{j}$ 
Where $C_{\text {diff }}$ is the concentration of diffusible hydrogen atoms per unit volume, $C_{\text {trap }}^{i}$ is the concentration of trapped hydrogen atom per unit volume and $i$ represents the number of traps (and trap sites may be of different types in the model such as reversible trap sites, irreversible trap sites, defect trap sites such as void trap sites, GND trap sites etc.), $N_{t}^{i}$ is the volume density of traps per unit volume, $n_{i}$ is the fractional trap occupancy levels for $N_{t}^{i}$ traps, $k_{i}$ is hydrogen entry/capture rate per trap, $t$ is time, $p_{i}$ is the hydrogen exit/release rate per -for irreversible traps the release rate is zero. $N_{t}^{G N D}$ isthe GND density of trap sites, $\rho_{G N D}^{a}$ is the average GND density $\left(\mathrm{m}^{-2}\right)$ where $\rho_{G N D}^{a}=\frac{1}{2 d} \sum_{j=1}^{m_{g b}} \frac{1}{s_{j}}=\frac{1}{b d} \sin \left(\frac{\theta_{j}}{2}\right) \cdot f_{j}[32,60]$. The quantity $\rho_{G N D}^{a}$ in any given grain boundary can be calculated through electron backscattering diffraction (EBSD) analysis using the GB misorientation angle and a single dislocation network is necessary to accommodate the GB misorientation [32,60,68-71]. Here, $r$ is the effective distance between dislocations, $N_{L}$ is the density of interstitial sites $\left(\mathrm{m}^{-3}\right)$ or the number of solvent lattice atoms per unit volume $\left(N_{L}=N_{A} / V_{M}\right.$, where $N_{A}$ is Avogadro's number and $V_{M}$ is the molar volume of the host metal lattice), $m_{g b}$ is the number of types of GBs studied, $b$ is Burgers vector, $d$ is the average grain size, $s_{j}$ is the distance between two dislocations in a GB misorientation of $\theta_{j}$ where from dislocation theory $s=\frac{b}{2} \sin \left(\frac{\theta}{2}\right)$ [44], $f_{j}$ is the fraction of GB with misorientation angle $\theta_{j}$ and the transition probabilities are expressed by an Arrhenius expression $\frac{k_{i}}{p_{i}}=\frac{1}{N_{L}} \exp \left(\frac{-E_{B}}{R T}\right)$ suitable for thermally activated processes.

\section{FE formulation of trap model:}

The finite element method discretises the unknown concentration in space and time as follows,

$C(\boldsymbol{x}, t) \approx \sum_{k=1}^{r_{\text {nodes }}} C_{k}(t) N_{k}(\boldsymbol{x})$

Where $C_{k}(t)$ is the concentration at $k^{\text {th }}$ node and $N_{k}(\mathbf{x}), k=1,2, \ldots, r_{\text {nodes }}$ are a set of nodal basis functions defined on the finite element mesh. It is assumed that the trap occupancy levels $n_{i}$ are constant within each element. This assumption leads to a discretisation as follows.

$n_{i}(\boldsymbol{x}, t) \approx \sum_{l=1}^{r_{\text {elements }}} n_{l, i}(t) M_{l}(\boldsymbol{x})$

Where $n_{l, i}$ is the trap occupancy level for $i$-type traps in the $l^{\text {th }}$ element, $M_{l}(x, t)$ is a function that is unity everywhere within the $l^{\text {th }}$ element and zero outside of it. This approach means the trap occupancy levels are discontinuous, but since their spatial derivatives do not appear in the equations above this is unlikely to cause numerical problems and any problems with the 
validity of sharply discontinuous results can be resolved by refinement of the mesh [64]. Because (a) the spatial derivatives of the trap occupancy levels do not appear in the equations above and (b) the trap occupancy levels can be regarded as derived quantities dependent on the solution variable (i.e. concentration) then the FE formulation does not need to solve a matrix of equations to update explicitly the values of the trap occupancy levels during each step. A detailed explanation can be found elsewhere [64].

Using the standard Galerkin approach and applying the discretisation described above, the full finite element formulation can be shown to be of the form

$\sum_{w=1}^{r_{\text {node }}} \int_{\Omega} N_{k} N_{w} \frac{\partial C}{\partial t}+\nabla N_{k} \cdot\left(D_{g b} C \cdot \nabla N_{w}\right) d \Omega+\sum_{w=1}^{r_{\text {node }}} \int_{\partial \Omega} N_{k}\left(D_{g b} C \cdot \nabla N_{w}\right) \cdot \mathbf{n} d S+$

$\int_{\Omega} N_{k}\left(N_{t}^{i} \sum_{l=1}^{r_{\text {element }}} \frac{\partial n_{l, 1}}{\partial t} M_{l}(x)\right) d \Omega=0, k=1,2,3 \ldots . r_{\text {node }}$

Where $N_{k}$ and $N_{w}$ are basis functions and $N_{t}^{i}$ is the trap density. $\Omega$ is the domain of the model, $\partial \Omega$ represents boundaries and $\mathbf{n}$ is the outward unit normal to a given boundary. In practice the boundary integral would be replaced by appropriate boundary conditions, using a Lagrange multiplier if necessary to account for fixed concentration conditions.

\section{UEL Trap Subroutine Implementation:}

The trap fluxes in equation (5) must depend on the local trap occupancy levels and the local hydrogen concentration. The hydrogen concentration is the main solution variable in the model where as the trap occupancy levels are a user-defined variable for which values must be calculated as the model runs. The user defined element (UEL) calculates and stores the trap occupancy levels at each time increment overlaying the existing ABAQUS hydrogen diffusion model using the same nodes so that the solution variable and user-defined variables are coupled using the same nodal points as shown in the sketch below. Here hydrogen diffusion elements (orange) and trap elements (black) use the same nodes (circles) and connectivity.
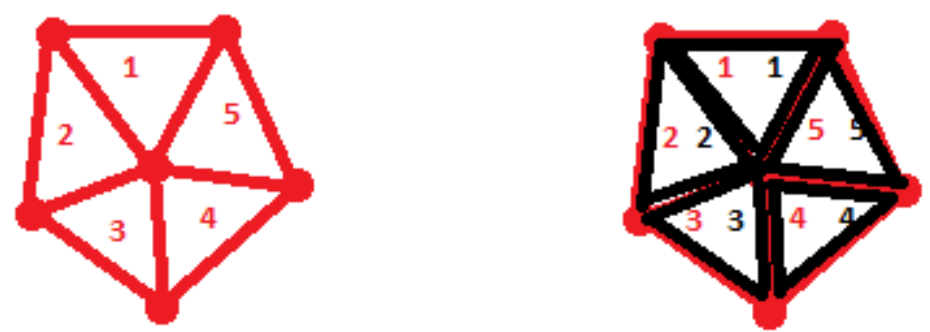

From above equation (11), it can be seen that the ABAQUS user element subroutine must generate values for

$\int_{E_{v}} N_{k}\left(N_{t}^{i} \frac{\partial n_{i}}{\partial t}\right) d \Omega$ 
Where $E_{v}$ is the trapping element, for all basis functions $\left(N_{k}\right)$ that correspond to the nodes making up $E_{v}$. These expressions have been evaluated using single-point numerical quadrature, since the basis function chosen for the trap occupancy level means that a higher order scheme would not improve the accuracy. Single point quadrature is also used to evaluate the contribution of the trapping term to the stiffness matrix, as required by Abaqus. The time discretisation has been implemented using the Crank-Nicolson method, rather than the more computationally expensive Backwards Euler method, since it is unlikely that stability problems will occur in this model. Constraints have been added to ensure that the trap occupancy level remains between 0 and 1. The MC simulations were used to assign the random distribution of crystal orientation and GB misorientation to generate reliable microstructural (i.e trap) properties and one coupled with FE code for hydrogen diffusion analysis.

\subsection{GB segregation of hydrogen for MC-FE model:}

Hydrogen induced intergranular cracking are due to the attainment of critical hydrogen concentration at GBs and segregation of hydrogen at GBs various depends on GB misorientation and GB type. The hydrogen segregates at GB determines the initiation of intergranular crack and the crack propagation path. So it is important to understand the segregation at GBs and the relation between hydrogen segregation at GBs and its type. The hydrogen segregation at GBs is measured using the segregation co-efficient as shown below. The GB hydrogen segregation coefficient $\left(S_{g b}\right)$ can be calculated based on the equation,

$S_{g b}=\frac{C_{g b}}{C_{L}}=\exp \left(\frac{-E_{B}}{R T}\right)$

where $E_{B}$ is the binding energy of a solute to an average GB sites.

The lattice and GB hydrogen concentration can be calculated based on the diffusible hydrogen concentration, $C_{H, d i f f}$ as shown in the below equation

$C_{H, \text { diff }}=C_{L}+C_{\mathrm{gb}, \mathrm{rev}}=n_{L} N_{L}+\sum_{i}^{q} n_{i, r} N_{g b, r}^{i}$

where $C_{L}=n_{L} N_{L}$ is the hydrogen concentration in lattice sites, $C_{\mathrm{gb}, \mathrm{rev}}$ is the hydrogen concentration of a reversible trap, $n_{i, r}$ is reversible GB trap occupancy level, $N_{g b, r}^{i}$ is the reversible trap density $\left(\mathrm{m}^{-3}\right)$ for a number, $\mathrm{q}$, of reversible traps.

$D_{\text {eff }}$ istheeffective diffusivity of hydrogen given by,

$D_{\text {eff }}=\left[\gamma \cdot a_{0}^{2} v \exp \left(\frac{\beta \Delta H}{R T_{m p}}\right)\right] \cdot \exp \left(\frac{-E_{a}}{R T}\right)$ 
where $\beta=\frac{d\left(\mu / \mu_{0}\right)}{d\left(T^{T} / T_{m p}\right)}$ and $\gamma \cdot a_{0}^{2} v \exp \left(\frac{\beta \Delta H}{R T_{m p}}\right)=D_{0}$ is a pre-factor. $E_{a}$ is the activation energy for diffusion $(\mathrm{kJ} / \mathrm{mol}) . D_{0}$ and $E_{a}$ can be calculated by using a $\log$ plot of calculated $D_{\text {eff }}$ over a range of temperatures, $\gamma$ is the geometric factor, $a_{0}$ is the jump distance, $v$ is the frequency with which the solute atom vibrates in the diffusion direction, $\Delta H$ is the activation enthalpy for diffusion, $T_{m p}$ is the melting point of the pure metal, $\mu$ is the elastic modulus of the pure metal, $\mu_{0}$ is the elastic modulus at zero degrees absolute, more details can be found in [51].

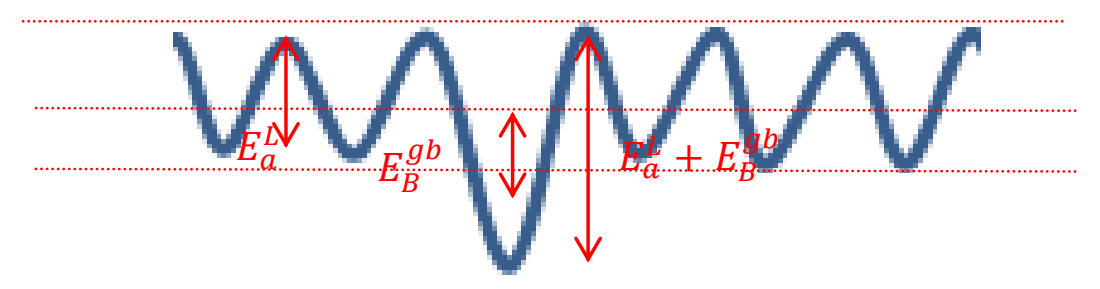

The sketch above shows the simple energy model for grain boundary traps [51], where $E_{a}^{L}$ is the activation energy for lattice diffusion $\operatorname{and} E_{B}^{g b}$ is the binding energy for a grain boundary trap.

At equilibrium condition, the effective diffusivity can be written as shown below

$D_{e f f}=\frac{D_{L}}{1+\frac{C_{g b}^{e}}{C_{L}}\left(1-\frac{C_{g b}^{e}}{N_{g b}^{e}}\right)}$

Note in the above equation $C_{g b}^{e}$ is the effectiveconcentration of all GB type sites and it is calculated as shown in equation (17). $N_{g b}^{e}$ is the effective GB type trap density and it can be calculated based on GB segregations as shown in equation (19) (i.e. in real polycrystalline microstructure materials there may be different types of possible GB sites based on type of the GB and GB misorientation). At certain orientation and planes, some lattice points of crystal 1 coincide exactly with some lattice points of crystal 2 as superstructure called as coincidence site lattice (CSL). The difference in trap occupancy levels in different types of GB is based on atomic arrangements and the difference in the binding energy for different GB types. For example in FCC nickel, the two distinct type of CSL GBs (i.e $\Sigma 3$ and $\Sigma 5$; $\Sigma 3$ type CSL GB have closed-packed interface structure with highly dense and $\Sigma 5$ type CSL GB have open-packed interfacial structure with less dense), the trap binding energy in the $\Sigma 3$ special CSL type GB is $0.02 \mathrm{eV}$ and in the $\Sigma 5$ general type GB is $0.2 \mathrm{eV}$, an order of magnitude difference in trap binding energies between two types of GB as reported by Mrovecet al[48, 72]. Changes in GB trap binding energy will change the hydrogen concentration in GB traps 
and hydrogen concentration will change due to the variation in GB trap densities according to equations (14). The changes in GB trap density vary according to the GB misorientation angle as shown in equation (8).

$C_{g b}^{e}=\sum_{i}^{n} n_{i} N_{g b}^{n}$

Where $n_{i}$ is the trap occupancy level for a number (superscript $n$ ) of GB type trap sites and $N_{g b}^{n}$ is the GB trap density for the different ' $n$ ' types of GBs.

The effective diffusivity can be written as shown below in the form of grain boundary segregation equation,

$$
D_{e f f}=\frac{D_{L}}{1+S_{g b}\left(1-\left(\frac{s_{g b} C_{L}}{N_{t}^{g b}}\right)\right)}
$$

And the effective grain boundary trap density for polycrystalline materials can be written as shown below in terms of GB segregations.

$N_{g b}^{e}=\frac{S_{g b} C_{L}}{1+\left(\frac{1}{s_{g b}}\right) \cdot\left(1-\frac{D_{L}}{D_{e f f}}\right)}$

Where $D_{\text {eff }}$ based on the segregation factor can be calculated using the modified Maxwell equation shown below [73-74],

$D_{e f f}=\frac{s_{g b} D_{g b}\left(\left(3-2 f_{g b}\right) D_{L}+2 s_{g b} D_{g b}\right)}{\left(1-D_{g b}+s_{g b} f_{g b}\right)\left(f_{g b} D_{L}+\left(3-f_{g b}\right) s_{g b} D_{g b}\right)}$

More detailed explanations of trap models including the simplified approach adopted by most workers and the UEL subroutine development and validation can be found elsewhere [11-12, 55-59, 63-65].

\subsection{COMPUTATIONAL MULTI-SCALE MULTI-PHASE MICROSTRUCTURAL MODEL}

The geometric multi-phase polycrystalline microstructural models are composed of three regions with two phases as shown in figure 2 for two different cases. Two particular cases are considered where the second phase is either GBAZ (Case 1) or an intergranular precipitate phase (Case 2). Figure 2(a) shows schematically the three different regions of the multi-phase polycrystalline microstructural model. The grain interior (GI) is represented by the red region and the thin green line bisecting the grains is the grain boundary line. The blue regions in figure 2 (a) and (b) are either:

- Grain boundary, Case 1 or 
- Phase boundary, Case 2.

For Case1,in figure 2 (a) and (b) the grain interior (GI) is represented by the red colour with low amounts of GNDs and the blue area is the GBAZ represented as a second region where high concentrations of GNDs are found. Figures 2(c), (d) and (e) show EBSD results for nickel polycrystalline material local misorientations, a close-up view of a few grains and a colour counter of local misorientation (which has been suggested as a measure of the GND density [67, 75, 76-78]). Further details concerning low GND in GIs and high GND in intergranular regions can be found elsewhere [35, 67, 75].

For Case 2, in figure 2(a) and (b) the grain interior (GI) is represented by the red colour with low amounts of GNDs and the blue region is a second region containing a double layer intergranular precipitate phase, either nano precipitates or micro precipitates. Figures 2(f) and (g) show the schematic view of random polygonal grains with different GBAZ/double layer intergranular interface thicknesses. Detailed descriptions of this multi-phase polycrystalline microstructural model for various GBAZ thicknesses can be found elsewhere [9, 25, 27-29, 66]. Previously reported that the GI of polycrystalline material where the atoms are in ideal lattice sites and GB regions where the atoms are relaxed away from ideal lattice sites [26]. In addition, it also previously reported by Schaefer et al. that the 2D schematic views of nanocrystalline material at the atomic scale with grain and grain boundary region separated by solid lines [31].

For Case 1, four different microstructures were computationally generated with double layer intergranular precipitate interfaces and various grain GBAZ thicknesses where the intergranular precipitate/GBAZ is embedded between irregular random polygonal crystalline grains. The domain size of the multi-phase polycrystalline microstructure for the four different GBAZ thicknesses is $4 \mathrm{~mm}^{2}$ and contains approximately 400 micro grains. For Case 2, two microstructure models with double layer intergranular precipitate interfaces containing (i) nano precipitates and (ii) micro precipitates were generated. The computationally generated multi-phase micro polycrystalline microstructures with various different GBAZ thicknesses, GBAZ networks/meshes plus EBSD results for micro polycrystalline microstructures are shown in Figure 3. Detailed information about the models of irregular random polygonal grains embedded with GBAZ/double layered intergranular interfaces can be found in Jothi et al. [8, 66]. The material properties used in simulations can be found below on the figures in the results section. An initial condition of zero hydrogen concentration in the material was used. Figure 4 shows the multi-phase micro polycrystalline 
model and boundary conditions for the hydrogen transport analysis in which hydrogen is transported at constant concentration (i.e. 1ppm) with fixed flux. The model was meshed with mesh sizes determined by mesh convergence tests (a typical mesh would contain approximately 110000 elements and 54000 nodes). Results of FE hydrogen transport simulations for various microstructures are discussed below.

\subsection{RESULTS AND DISCUSSION}

\subsection{The effect of microprecipitate and nanoprecipitate double layer intergranular interfaces on hydrogen transport:}

Two different multi-phase polycrystalline microstructural models, one with micro precipitate double layer intergranular interfaces (MPDII) and another with nanoprecipitate double layer intergranular interfaces (NPDII) have been used to numerically investigate the effect of these interfaces on hydrogen diffusion in polycrystalline materials. The surface area was the main difference between MPDII and NPDII. The first multi-phase polycrystalline microstructural model was computationally generated with $10 \mathrm{~nm}$ thick NPDII interfaces based on experimental TEM observations of NPDII in polycrystalline material with thicknesses between 10-15nm [42-43]. A second multi-phase polycrystalline microstructural model was investigated with $1 \mu \mathrm{m}$ thick MPDII interfaces based on SEM observations of precipitate hardened nickel based super alloy polycrystalline material as shown in Figure1.In both models the grain size, shape and structure, the domain size and the hydrogen diffusivity between precipitate interface and GI are the same.

Figures 5(a) and (b) show the results of FE hydrogen diffusion analyses for MPDII and NPDII interfaces respectively. Figure5(c) shows the normalized hydrogen concentration over time (log scale) at a fixed distance, $300 \mu \mathrm{m}$, from the top face of the computational grid for both MPDII and NPDII interfaces. The results show faster effective diffusion of hydrogen in polycrystalline material with micro precipitate intergranular interfaces compared to the nano precipitate intergranular interface cases. This is due to the larger surface area of the intergranular micro precipitate region. (It should be noted that the effective diffusion for the micro precipitate intergranular would be lower than the nanoprecipitate intergranular case if hydrogen diffusion in the precipitate phase were lower than the GI phase). Sjoberg et al reported that hydrogen trapping takes place in secondary phases in nickel based super alloys $[21,26]$. So to investigate hydrogen trapping effects, figures 5(d) and (e), the ABAQUS trap model UEL subroutine described in section2.2 was implemented on the intergranular 
precipitate phases as shown in figure 5(f). The results show the effective diffusion of hydrogen for microstructures with MPDII and NPDII interfaces are hindered by trapping compared to the non-trap model due to segregation of hydrogen into the precipitate intergranular interfaces. The effective diffusion parameter may vary depending on specific intergranular precipitate diffusion properties such as diffusivity and volume fraction. Figure $5(\mathrm{~g})$ shows the normalized hydrogen concentration for the MPDII case with and without the trap model at two different positions (i.e position $\mathrm{x} 1$ and $\mathrm{x} 2$ as shown in figure 5 (e)). This strongly suggests that for applications such as hydrogen storage and polycrystalline energy materials, and where hydrogen embrittlement is important, the role of intergranular precipitate phases should be taken into account.

\subsection{Effect of GBAZ on hydrogen transport in polycrystalline microstructures:}

Four different microstructure non-trap models with four different GBAZ thicknesses were modelled to investigate hydrogen transport in these microstructures. Figures 6 shows the computational hydrogen transport analysis results for the four different GBAZ cases after 1 hour and 1 day (i.e figures6(a), (b), (c) and (d) are for GBAZ thicknesses of 0.005,0.05, 0.5 and $5 \mu \mathrm{m}$ respectively). These simulations all used the same underlying grain structure. They differ only in the proportion of GBAZ assigned at the grain boundary regions. Larger proportions of GBAZ can be seen in figure 3 as thicker lines surrounding the grains. The simulations used values characteristic of typical GBAZ thicknesses ranging from $0.005 \mu \mathrm{m}$ to $5 \mu \mathrm{m}$ (figures6(a), (b), (c) and (d) are for GBAZ thicknesses of 0.005,0.05, 0.5 and $5 \mu \mathrm{m}$ respectively). Thus GBAZ thicknesses spread over four orders of magnitude are simulated. The results show the influences of the GBAZ thickness on hydrogen transport at $25{ }^{\circ} \mathrm{C}$ after 1 hour (central column Figure 6) and 1 day (right column Figure 6). Hydrogen diffusion for microstructures with smaller GBAZ thickness/surface area is slower when compared to larger GBAZ thicknesses/surface areas. An order of magnitude increase in GBAZ thickness increases the effective hydrogen diffusion distance more than twice and a four orders of magnitude increase in GBAZ thickness increases the effective hydrogen diffusion distance more than eight times. These results suggest that decreasing grain size will promote bulk diffusion of hydrogen due to the increase in grain boundary surface area in polycrystalline nickel. The results also show the heterogeneous distribution of hydrogen in bulk polycrystalline material along GBAZ and grain interior after a time period of 1 hour. This suggests that intergranular softening of polycrystalline material due to the segregation of atomic hydrogen along the intergranular microstructure may lead to intergranular hydrogen 
embrittlement (as opposed to transgranular hydrogen embrittlement) at shorter times, before the system attains a more steady state. This depends on the particular intergranular diffusion properties but could be an important consideration when considering hydrogen embrittlement effects. Figures 7(a) and (b) show the predicted hydrogen concentration in polycrystalline material with various GBAZ thicknesses. This shows that the hydrogen concentration profile attains steady state earlier for higher relative fractions of grain boundary polycrystalline material. A number of studies have already reported that the relative fraction of grain boundary in nano polycrystalline nickel is significantly larger than the case of micro polycrystalline nickel while observing faster diffusion in the nano polycrystalline case $[9,11$, 22]. These observations are in agreement with the model prediction that bulk diffusion in nano polycrystalline nickel will be faster than that for the micro polycrystalline case.

\subsection{Influences of intergranular grain boundary network on hydrogen diffusion in multi- phase polycrystalline material:}

Figure 8 shows the normalized hydrogen concentration along the normalized distance along a path from the hydrogen source to the hydrogen sink for various GBAZ thicknesses after diffusion times of one hour and one day. Hydrogen diffusion is heterogeneous between grain and grain boundary in the polycrystalline material becoming more homogenous with decreasing grain boundary thickness and/or increasing time. Even though the interior grain and grain boundary diffusion coefficients are same for all GBAZ thicknesses simulated the model predicts less diffusion of hydrogen for smaller GBAZ thicknesses. This enhanced hydrogen diffusion for larger GBAZ thicknesses is due to the increased contribution of the intergranular grain boundary density as shown in figure 8 . The increased density of grain boundary in smaller grained polycrystalline materials greatly increases the hydrogen diffusion along the grain boundary thus increasing the effective bulk hydrogen diffusion. These results show the significant influences of intergranular grain boundaries on the bulk hydrogen diffusion process in micropolycrystalline and nanopolycrystalline materials.

\subsection{Segregation effects of hydrogen at intergranular grain boundary regions in micro and nano polycrystalline material}

For this study three different computationally generated multi-phase polycrystalline model (i.e non-trap based model) have been developed with different grain sizes (one with nano or ultra-fine grains, another with fine micro grains and another with coarse micro grains) to 
investigate their influence on segregation of hydrogen in the intergranular GB region. Figure 9 (a) shows the results of hydrogen diffusion in nano polycrystalline material and micro polycrystalline material after one second (i.e Nanopolycrystalline model with nano grains are meshed with very fine mesh and in order to run the analysis for one hour it need high computational time and resources, so the nanopolycrystalline model were analysed for one second). It clearly shows that the hydrogen diffusion in nano polycrystalline material is much faster than the micro polycrystalline material. Figure 9 (b) and (c) shows the results of hydrogen diffusion analyses in micro polycrystalline materials with two different GBAZ thicknesses after 1 hour. It shows that the increase in grain boundary thickness increase the effective diffusion of hydrogen in the polycrystalline material. It also clearly shows that hydrogen diffusion between the grain interior and grain boundary is inhomogeneous and diffusion of hydrogen in the intergranular grain boundary network is higher than diffusion within the polygonal grains. Hydrogen flux increases along grain boundaries and hydrogen accumulates more in the grain boundaries than inside grains, as shown in Figure 9. These results also show that the inhomogeneous diffusion and segregation of hydrogen along grain boundaries becomes more homogeneous when moving towards steady state. Hydrogen diffusion in nano polycrystalline material becomes homogenous much earlier compared to the fine and coarse micro polycrystalline materials. The micro polycrystalline materials need more time to attain steady state. This suggests that the hydrogenated fractures occurring before steady state in micro polycrystalline materials may be intergranular due to embrittlement along grain boundaries.

\subsection{Effects of hydrogen segregation factor, GND trapping density, lattice hydrogen concentration on effective diffusivity of hydrogen in polycrystalline materials:}

Finally, by using a Monte Carlo model the relationships between hydrogen segregation factor, hydrogen concentration in lattice sites and density of GND trapping sites in the polycrystalline material on the effective diffusivity of hydrogen in polycrystalline material were investigated. MC statistical models aim to develop a physically realistic insight into the spatial aspects of crystal lattice orientations and GB misorientations during microstructure evolution, quantify the effects of anisotropic GB misorientation properties, and quantify the effects of GND densities for hydrogen trapping sites. For all the GB misorientation simulations a distribution of $10^{4}$ sites was employed. The MC simulations were also performed to calculate effective diffusivities of hydrogen by varying the density of GND 
trapping sites and by fixing the hydrogen concentration in lattice sites in order to investigate the segregation factors of atomic hydrogen indifferent GB misorientations. The averages of $10^{4} \mathrm{MC}$ samples of atomic lattice concentration of hydrogen, density of GND trapping sites and segregation factors of atomic hydrogen in GB misorientations were used to calculate effective diffusivities of hydrogen. The results of the MC simulation and the statistical sample fluctuation data used can be found in figure 10. Note the parameter distribution limits used for this MC simulation are the experimental data reported for a polycrystalline nickel material [32, 37, 60]. Figures 10(a), (b) and (c) show the distribution of the hydrogen segregation factor, the atomic concentration of hydrogen in the lattice and the density of GND trapping sites respectively for various GB misorientations. Figure 10 (d) shows the distribution of calculated effective diffusivities of hydrogen in the polycrystalline material. It also shows that more than half of the calculated effective diffusivities are between $2 \times 10^{-16}$ and $8 \times 10^{-16} \mathrm{~m}^{2} \mathrm{~s}^{-1}$, that is, the random sampling in (i) segregation factor between 0 to 100 , (ii) lattice hydrogen concentration between 0 to $1 \mathrm{ppm}$ and (iii) GND density trapping sites between $10^{26}$ to $10^{27}$ $\left(\mathrm{m}^{-3}\right)$, reduces the effective diffusivity of hydrogen in polycrystalline material by two orders of magnitude compared to the lattice diffusivity of hydrogen. One third of the calculated effective diffusivities are between $8 \times 10^{-16}$ and12 $\times 10^{-16} \mathrm{~m}^{2} \mathrm{~s}^{-1}$. Figures $10(\mathrm{e})$ and (f) show the MC simulation results of the effective diffusivity of hydrogen distribution in polycrystalline material with respect to the distribution of the densities of GND trapping sites and lattice hydrogen concentration respectively. Figure $10(\mathrm{~g})$ shows the distribution of hydrogen segregation factor for respective densities of GND trapping sites. These show that even small variations in any one of these parameters may alter the effective diffusivity of hydrogen significantly. Figure 10(h) shows the distribution of effective hydrogen diffusivities against respective hydrogen segregation factors. In figure $10(\mathrm{~h})$, in region I, the hydrogen segregation factor varies from zero to 10 and the effective hydrogen diffusivity varies significantly. Region II is a transition region where the effective diffusivity is moving towards steady state. Steady state is attained in region III. The model suggests that a hydrogen segregation factor of about 10 is a critical value, below this value the effective hydrogen diffusivity of polycrystalline material is affected significantly more when compared to segregation factor values greater than 40. Calculated trap densities based on the statistical distribution of GB misorientations are shown in figure 10(i). Figures 10(j) and (k) show the grain boundary trapping site binding energies and respective segregation factor and the distribution of the binding energies. Finally, figure 11 shows a novel one way coupling of MC simulation with 
the FE multi-scale multi-phase continuum trap model where the hydrogen segregation is based on GB misorientation and this novel coupled model has been used to calculate the local GB/Grain scale segregations factors. In this latter model, MC routines were integrated into the commercial finite element software ABAQUS using PYTHON scripting. The GND densities were generated by MC simulation as inputs for the FE model based on Euler angles. $10^{4}$ Euler angles were simulated randomly using the MC model for each grain. The algorithm converts the 20000 Euler angles between two neighboring grains to develop $10000 \mathrm{~GB}$ misorientationsin the form of a Gaussian distribution. The average calculated GB misorientations are used in the GBAZ regions in the FE multi-phase continuum trap model to obtain both low angle grain boundaries (LAGBs) and high angle grain boundaries (HAGBs). It should be stressed that in case of HAGBs the assumption of one GND was still used although the model has the potential to be further developed to deal with multiple GNDs. The coupled MC-FE multi-scale multi-phase continuum trap model is a novel technique to potentially provide physically-realistic crystal lattice orientation and GB misorientation data for simulations of local grain/GB nano/micro scale hydrogen distributions in clean energy power source fuel carrier/storage materials and to study IHE in polycrystalline materials.

This section clearly shows the effects of atomic and microstructural parameters affecting the effective diffusion of hydrogen in polycrystalline material. This reinforces the idea that these microstructural parameters should be taken into account when calculating effective diffusivities. Computational FE modeler should aware that these microstructural parameters plays important role in the effective diffusivities and microstructural parameters based effective diffusivities should be used when modelling macro scale components for studying hydrogen induced intergranular and transgranular embrittlement problems in polycrystalline materials. In this study, several multi-scale models are analyzed and huge amount of data and data sets are collected through multi-scale modelling in addition with experimentation to understand the effects of hydrogen atoms on intergrangular and transgranular fracture in polycrystalline material. All these data and data sets need to be properly stored, carefully handle and transferred in order to investigate hydrogen embrittlement and hydrogen induced cracking using data-driven approach.

\subsection{CONCLUSION}

This paper has described models of multi-scale multi-phase polycrystalline microstructures to investigate the hydrogen transport, segregation and trapping in double layer 
intergranular micro-precipitate and nano precipitate interfaces, varying thickness GBAZs, micropolycrystalline and nanopolycrystalline materials and the effect of GNDs on hydrogen segregation. A trapping model has been developed as a UEL subroutine for ABAQUS software using FORTRAN to study hydrogen trapping in multi-phase polycrystalline material. A coupled MC-FE multi-scale multi-phase continuum trap model has also been developed to study the hydrogen transport and segregation. The results of the proposed model demonstrate that the random micro precipitate/nano precipitate are high/low diffusion paths of hydrogen at room temperature depending on the diffusivity along the second intergranular precipitate phase which will affect the effective transport and segregation of hydrogen. Higher densities of random GBAZ lead to faster diffusion of hydrogen in polycrystalline material when compared to lower densities of GBAZ. The consequence of this is that the time taken to attain steady state is much less in larger GBAZ thickness polycrystalline material due to the higher relative fraction of GBAZ surface area.

Hydrogen diffusion and segregation in micropolycrystalline and nanopolycrystalline material between grains and grain boundaries are inhomogeneous and much greater hydrogen concentrations are accumulated in grain boundaries than grains. Hydrogen diffusion and segregation is greater in nanopolycrystalline material due to the higher density of grain boundaries. A novel technique has been proposed to predict physically-realistic values for crystal lattice orientations, GB misorientations and GND densities by coupling MC simulation with an FE multi-scale multi-phase continuum trap model. This proposed model shows that hydrogen transport and effective hydrogen diffusion properties in multi-phase polycrystalline material are highly sensitive to physical microstructural factors such as second phase intergranular precipitate interface, GB thickness, density of grain boundaries, GND trapping density and hydrogen segregation factor. The results also show inhomogeneous hydrogen diffusion and segregation mechanisms between complex irregular random polygonal grains and random GBAZs, at both micro and nano scale. This could be used as guidance for experimental investigations in order to understand the different modes of hydrogen induced embrittlement in practical applications. Workers developing hydrogen storage and energy polycrystalline materials and investigating hydrogen embrittlement in these materials should take features such as intergranular precipitate phases, GBAZs and GNDs into account.

Future work will include incorporation of micro and nano clustered grains into the coupled MC-FE trap model and extend the model to investigate (i) stress, temperature and strain assisted hydrogen distribution/segregations and(ii) localized plasticity and hydrogen 
assisted cracking mechanisms in hydrogen embrittlement problems for clean energy power source polycrystalline materials.

\section{ACKNOWLEDGEMENTS}

This work was supported by European Union under the Seventh Framework programme, project grand number 263335MultiHy (Multi-scale Modelling of Hydrogen Embrittlement in crystalline materials).

\section{REFERENCES}

1. Arantes, D.R., Huang, X.Y., Marte, C. and Kirchheim, R., ActaMetall. Mater., 41, No. 11, (1993)3215-3222.

2. Windle, A.H., Smith,G.C., Metal Sci. J. 12(1968) 187-191.

3. Kurkela, M.,Latanision, R.M., Scripta Metal. 13(1979)927-932.

4. Turnbull, A.,Corrosion Science 34 (1993)921-960.

5. Lynch, S.P., Practical Failure Analysis 3 (2003) 33-42.

6. Xu, J., Sun, X.K., Liu, Q.Q.,Chen, W.X., Metall, Mater. Trans. 25A, (1994) 539-544.

7. Byun, T.S.,Farrell, K.,J. Nuclear Materials318 (2003) 292-299

8. Liu, L.,Tanaka, K. Hirose, A., Kobayashi, K.F.,Science and Technology of Advanced Materials3 (2002) 335-344.

9. Jothi, S.,Croft, T.N.,Brown, S.G.R.,Int. J.Hydrogen Energy39 (2014)20671-20688.

10. Jothi, S., Croft, T.N., Brown, S.G.R.,ICHP2014 Conf.Proc., Feb(2014) Fukuoka, Japan.

11. Turnbull, A., Carroll,M.W. and Ferriss, D.H.,ActaMetal. 37 (1989) 2039-2046.

12. McNabb, A., Foster, P.K.,Trans Metall. Soc. A.I.M.E. 227 (1963) 618-627.

13. Reese, E.D., von Bestenbostel, W., Sebald, T., Paronis, G.,Vanelli, D., Muller, Y.,JOM 66 (2014), 368-1376.

14. Sjoberg, G.,Cornu, D.,"Hydrogen Embrittlement of Cast Alloy 718 Effects of Homogenization, Grain Size and $\delta$-phase", in "Superalloys 718, 625, 706 and various derivatives", The Minerals, Metals \& Materials Society,ed.Loria, E.A., (2001) 679690.

15. Bernstein, I.M., "The role of Hydrogen: Is the Story any Clearer?",in "Hydrogen Effects in Materials", eds. Thompson,A.W.,Moody, N.R., The Minerals, Metals \& Materials Society (1996) 3-11.

16. Fritzmeier,L.G., Chandler, W.T.,"Hydrogen Embrittlement-Rocket Engine Applications", in "Superalloys, Supercomposites and Superceramics", eds. Tien, J.K., Caulfield, T., Materials Science Series, Academic Press, Inc., (1989) 491-524. 
17. Jewett,R.P.,Halchak, J.A.,"The use of alloy 718 in the space shuttle main engine"in "Superalloys 718, 625, 706 and various derivatives", The Minerals, Metals \& Materials Society,ed.Loria, E.A.,(1991) pp.749-760.

18. Fukuyama, S., Yokogawa, K.,"Effect of heat treatments on hydrogen environment embrittlement of alloy 718" in "Superalloys 718, 625, 706 and various derivatives", The Minerals, Metals \& Materials Society,ed.Loria, E.A.,(1994) 807-816.

19. Pound, B.G., Acta Metall. Mater., 38 (1990) 2373-2381.

20. Marte, C., Kirchheim, R., Scripta Mat., 37, No.8 (1997) 1171-1175.

21. Richard, P.G. and Brain, P.S., Gaseous Hydrogen Embrittlement of Materials in Energy TechnologiesVolume2: Mechanisms, Modelling and Future Developments, (2012)Woodhead Publishing, Cambridge.

22. Harris,T.M., Latanision, R.M., Met. Trans. A, 22A (1991) 351-355.

23. Brass, A.M., Chanfreau, A., ActaMater., 44, No.9(1996) 3823-3831.

24. Yao, J. and Meguid, G.A., Int. J. Hydrogen Energy, 22, No.10/11 (1997) 1021-1026.

25. Jothi, S., Croft, T.N., Brown, S.G.R.,de Souza Neto, E., Composite Structures, 108 (2014) 555-564.

26. Meyers, M.A., Mishra, A., Benson, D.J., Progress in Materials Science, 51 (2006) 427-556.

27. Paggi, M., Wriggers, P, Comp. Mat. Sci., 50 (2011) 1634-1643.

28. Gleiter, H., Prog.in Mat. Sci., 33 (1989) 223-315.

29. Meyers, M.A., Ashworth, E., Phil. Mag. A, 46, No.5 (1982) 737-759.

30. Benson, D.J., Fu, H.H., Meyers, M.A., Mat. Sci. and Eng., A319-321 (2001) 84-861.

31. Schaefer, H.-E., Wurschum,R., Birringer,R., and Gleiter, R., Phys.Rev. B, 38, No.14 (1988) 9545-9554.

32. Oudriss, A., Creus,J., Bouhattate,J., Conforto,E., Berziou,C., Savall,C., Feaugas,X., ActaMater., 60 (2012) 6814-6828.

33. Jothi, S., "Multiscale modeling and experimentation of hydrogen embrittlement in aerospace materials", PhD Thesis, Swansea University, UK,(2015).

34. Jothi, S., Croft, T.N., Brown, S.G.R.,de Souza Neto, E., $12^{\text {th }}$ International conference on Structural Integrity Assessment. May 2013, Manchester, UK.

35. Jothi, S., Croft, T.N., Brown, S.G.R.,"EU FP7 Multi-ScaleModelling Hydrogen Embrittlement- MultiHy", Swansea University Report (2014).

36. Reese, E.D., von Bestenbostel, W., Sebald, T., Paronis, G.,Vanelli, D., Muller, Y.,JOM 66 (2014), 368-1376.

37. Tsuru,T., Latanision, R.M., ScriptaMetall, 16 (1982) 575-578.

38. Watanabe, T.,Journal de Physique, 46 C4 (1985) 555-564.

39. Jothi, S., Croft, T.N., Brown, S.G.R.,J. Alloys and Compounds, 645 (2015) S500S504.

40. Jothi, S., Croft, T.N., Brown, S.G.R.,Int. J.Hydrogen Energy 40 (2015) 2882-2889. 
41. Jothi, S., Winzer, N., Croft, T.N., Brown, S.G.R.,J. Alloys and Compounds, 645 (2015) S247-S251.

42. Raabe, D., Herbig, M., Sandlobes, S., Li, Y., Tytko, D.,Kuzmina, M., Ponge, D., Choi, P-P., Current opinion in Solid State and Material Science 18 (2014)253-261.

43. Raabe, D., Sandlobes, S.,Millan, J., Ponge, D., Assadi, H.,Herbig, M.,Choi, P-P.,Acta Mater. 61 (2013) 6132-6152.

44. Turnbull, D., Hoffman, R.E., Acta Metall., 2 (1954)419-426.

45. Canon, R.F.,Stark, J.P., J. App.Phys., 40 (1969)4366-4373.

46. Lejcek, P., "Grain Boundary Segregation in Metals", Springer Series in Materials Science 136 (2010).

47. Mills, W.J., Lebo, M.R., Kearns, J.J.,Metall. Mater. Trans. A 30 (1999)1579-1596.

48. Di Stefano, D., Mrovec,M., Elasser, C., Acta Mater., 98 (2015) 306-312.

49. Haftbaradaran, H., Song, J., Curtin, W.A., Geo, H., J.Power Sources 196 (2011)361370.

50. Song, J., Curtin, W.A., Nature Mat. 12 (2013)145-151.

51. Harris, T.M., "Hydrogen Diffusion and Trapping in Electrodeposited Nickel", PhD Thesis, Massachusetts Institute of Technology, USA,(1989).

52. Lassila,D.H.,Birnbaum, H.K, Acta Metall., 34 (1986)1237-1243.

53. McLean, D., "Grain Boundaries in Metals", Oxford University Press (1957).

54. Lee, S-M., Lee, J-Y., Met. Trans. A, 17A (1986)181-187.

55. Iino, M., Acta Metall. 30 (1982) 367-375.

56. Iino, M., Acta Metall. 30 (1982) 377-383.

57. Leblond, J.B., Dubois, D., Acta Metall. 31 (1983)1459-1469.

58. Leblond, J.B., Dubois, D., Acta Metall.31 (1983)1471-1478.

59. Oriani, R.A.,Acta Metall.18 (1970)147-157.

60. Oudriss, A.,Creus, J.,Bouhattate, J.,Savall, C.,Peraudeau,B.,Feaugas, X.,Scripta Mater. 66 (2012) 37-40.

61. Louthan Jr., M.R.,Donovan, J.A.,Caskey Jr., G.R.,Acta Metall. 23 (1975) 745-749.

62. Ladna, B., Birnbaum, H.K.,Acta Metall. 35 (1987) 1775-1778.

63. ABAQUS Version 6.11. User's Manual, DassaultSystemes, (2011).

64. Wright,L., Turnbull, A., "EU FP7 Multi-ScaleModelling Hydrogen EmbrittlementMultiHy", National Physical LaboratoryReport (2013).

65. Jothi, S., Croft, T.N., Brown, S.G.R., de Souza Neto, E.,"EU FP7 Multi-Scale Modelling Hydrogen Embrittlement- MultiHy", Swansea University Report (2013).

66. Jothi, S., Croft, T.N., Brown, S.G.R., Composites Part B: Engineering, 75 (2015) 104118.

67. Birosca, S.,Di Gioacchino, F.,Stekovic, S., Hardy, M.,Acta Mater. 74 (2014) 110-124.

68. Ashby, M.F.,Phil. Mag. 21 (1970) 399-424.

69. Pesicka, J.,Dronhofer, A.,Eggeler, G.,Mat. Sci. Eng. A 387-389 (2004) 176-180.

70. Nye, J.F.,Acta Metall. 1 (1953) 153-162.

71. Mandal, D.,Baker, I.,Scripta Metall. Mater. 33 (1995) 831-836.

72. Mrovec, M., Stefano,D.D., Elsasser, C., Nazarov, R., Hickel, T., "Hydrogen embrittlement: Multi-scale modelling and measurement - What is the impact?" Workshop, 6-8 October 2014, National Physical Laboratory, Teddington,UK.

73. Kalnin, J.R., Kotomin, E.A.,Maier, J.,J. Phys. Chem. Solids, 63 (2002)449-456.

74. Belova,I.V., Murch, G.E., J.Metastable and NanocrystallineMaterials, 49 (2004)2534. 
75. Raabe, D.,Zhao, Z., Park, S-J., Roters, F., Acta Mater. 50 (2002) 421-440.

76. Brewer, L.N., Field, D.P., Merriman, C.C., "Mapping and Assessing Plastic Deformation using EBSD", in"Electron Backscattering Diffraction in Material Science", eds. Schwartz, A.J., Kumar, M., Adams, B.L., Field, D.P., New York, Springer Science + Business Media (2000).

77. Koyama, M.K., Tasan, C.C., Akiyama, E., Tsuzaji, K., Raabe, D., Acta Mater. 70 (2014)174-187.

78. Koyama, M.K., Springer, H., Merzlikin, S.V., Tsuzaki, K., Akiyama, E., Raabe, D., Int. J.Hydrogen Energy 39 (2014)4634-4646. 


\section{List of Figure Captions:}

Figure 1: Intergranular double layer micro precipitates in nickel based polycrystalline superalloy used in space shuttle combustion chamber (NB precipitate hardened) and an atom probe study showing the segregation of phosphorus along a grain boundary in nickel based superalloy (Atom probe studies carried out at Chalmers University, Gothenburg, Sweden with Dr. Mattias Thuvander).

Figure 2: Schematic view of polycrystalline microstructure with double layer intergranular interface. (a) and (b) show the schematic view of polycrystalline material with double layer intergranular interfaces with different GBAZ thicknesses. (c) EBSD result showing the local misorientation in nickel microstructure, (d) shows the close view of local misorientations; (e) shows the local misorientation colour counters. (f) and (g) show the close up view of the computationally simulated multi-phase polycrystalline microstructure model with two different double layer intergranular interface thickness.

Figure 3: Computationally generated multi-phase micro polycrystalline microstructures with (a) different GBAZ and (b) double layered intergranular interface networks with different thickness and (c) EBSD analysis of micro polycrystalline microstructure.

Figure 4: Microstructure and boundary conditions (hydrogen source at top face and hydrogen sink at bottom face) of the model.

Figure 5: Hydrogen distribution and segregation with and without trapping for NPDII and MPDII interfaces in multi-phase polycrystalline materials. (NB hydrogen diffusivity in MPDII and NPDII is $4 \times 10^{-12} \mathrm{~m}^{2} \mathrm{~s}^{-1}$; Diffusivity in GI for both models is $9 \times 10^{-14} \mathrm{~m}^{2} \mathrm{~s}^{-1}$; elapsed time is $10^{4} \mathrm{~s}$ ). Colour contours are the same for all results. (c) Shows the normalized hydrogen concentration of multi-phase polycrystalline microstructure for NPDII and MPDII interfaces with and without the trap model. (f) The red color shows the precipitate double layer intergranular interface where the trap elements are implemented; (g) shows the normalized hydrogen concentration for the MPDII interface model with and without the trap model at two different positions.

Figure 6: Contours of hydrogen concentration in a computationally simulated polycrystalline material for increasing GBAZ thicknesses (a), (b), (c) and (d) are for GBAZ thicknesses of $0.005,0.05,0.5$ and $5 \mu \mathrm{m}$ respectively, after 1 hour (middle) and 1 day (right). Note: GI and GBAZ diffusivities used are $9 \times 10^{-14}$ and $4 \times 10^{-12} \mathrm{~m}^{2} \mathrm{~s}^{-1}$ respectively used in all simulations.

Figure 7: Normalized hydrogen concentration of polycrystalline microstructures with four different GBAZ thicknesses, $0.005,0.05,0.5$ and $5 \mu \mathrm{m}$, at two different positions. (a) $70 \mu \mathrm{m}$ and (b) $200 \mu \mathrm{m}$ from the top surface of the computational grid.

Figure 8: Normalized hydrogen concentration along the vertical path shown above in the polycrystalline material for GBAZ thicknesses of 0.05 (blue), 0.5 (green) and 5 (red) $\mu \mathrm{m}$ after (i) one hour (solid lines) and (b) one day (dotted lines).

Figure 9. Segregations/Accumulation of hydrogen along intergranular grain boundaries and inhomogeneous hydrogen diffusion processes along grain boundaries and within grains for (a) nano polycrystalline material with nano scale grains after one second and micro polycrystalline material after one second, (b) and (c) micro polycrystalline material with two 
different GBAZ thicknesses after 1 hour. Note: red dotted lines show the Segregations/accumulation of hydrogen in grain boundaries and blue dotted lines show the hydrogen concentration in grains). (Note: GBAZ diffusivity $=4 \times 10^{-12} \mathrm{~m}^{2} \mathrm{~s}^{-1}$ and GI diffusivity $\left.=9 \times 10^{-14} \mathrm{~m}^{2} \mathrm{~s}^{-1}\right)$. Note: Colour contours are same for all results).

Figure 10: Monte Carlo simulation results of effective diffusivity ( $D_{\text {eff }}$ ) for 10000 sample distributions assuming a lattice concentration $\left(\mathrm{C}_{\mathrm{L}}\right)$ from 0 to $1 \mathrm{ppm}$, density of trapping sites based on grain boundary misorientation $\left(\mathbf{N}_{\mathbf{t}}^{\mathbf{G N D}}\right)$ from $10^{26}$ to $10^{27}\left(\mathrm{~m}^{-3}\right)$, segregation factors (S) from 0 to 100 and a lattice diffusivity of $3.52 \times 10^{-14} \mathrm{~m}^{2} \mathrm{~s}^{-1}$. (a) distribution of segregation factors, (b) distribution of lattice concentration of hydrogen, (c) density of trapping sites based on grain boundary misorientation, (d) effective diffusion of hydrogen in polycrystalline material, its closed view and data used for statistical samples floatation's, (e) effective diffusivity of hydrogen as a function of density of trapping sites, (f) effective diffusivity of hydrogen as a function of lattice hydrogen concentration, (g) segregation factor distribution as a function of density of trapping sites, (h) effective diffusivity as a function of segregation factors, (i) statistical distribution of GB misorientations, (j) segregation factors versus average grain boundary trapping sites binding energy (increasing segregation factor with increasing $\mathbf{E}_{\mathbf{B}}^{\mathbf{g b}}$ ) and (k) Shows the distribution of average grain boundary trapping sites binding energy.

Figure 11: shows the hydrogen segregations results of the Monte Carlo simulation coupled with FE multi-scale multiphase continuum trap model based on (a) GB misorientations and (b) without GB misorientation and its segregations factors (S). 


\section{MULTI-PHASE MODELLING OF INTERGRANGULAR HYDROGEN SEGREGATION/TRAPPING FOR HYDROGEN EMBRITTLEMENT}

${ }^{*}$ S.Jothi ${ }^{1}$, T.N. Croft ${ }^{1}$, L. Wright ${ }^{2}$, A. Turnbull ${ }^{2}$ and S.G.R.Brown ${ }^{1}$

${ }^{1}$ College of Engineering, Swansea University, Singleton Park, Swansea, SA2 8PP, UK.

${ }^{2}$ National Physical Laboratory, Teddington, Middlesex TW11 0LW, UK.

*s.jothi@swansea.ac.uk

\section{ALL FIGURES:}

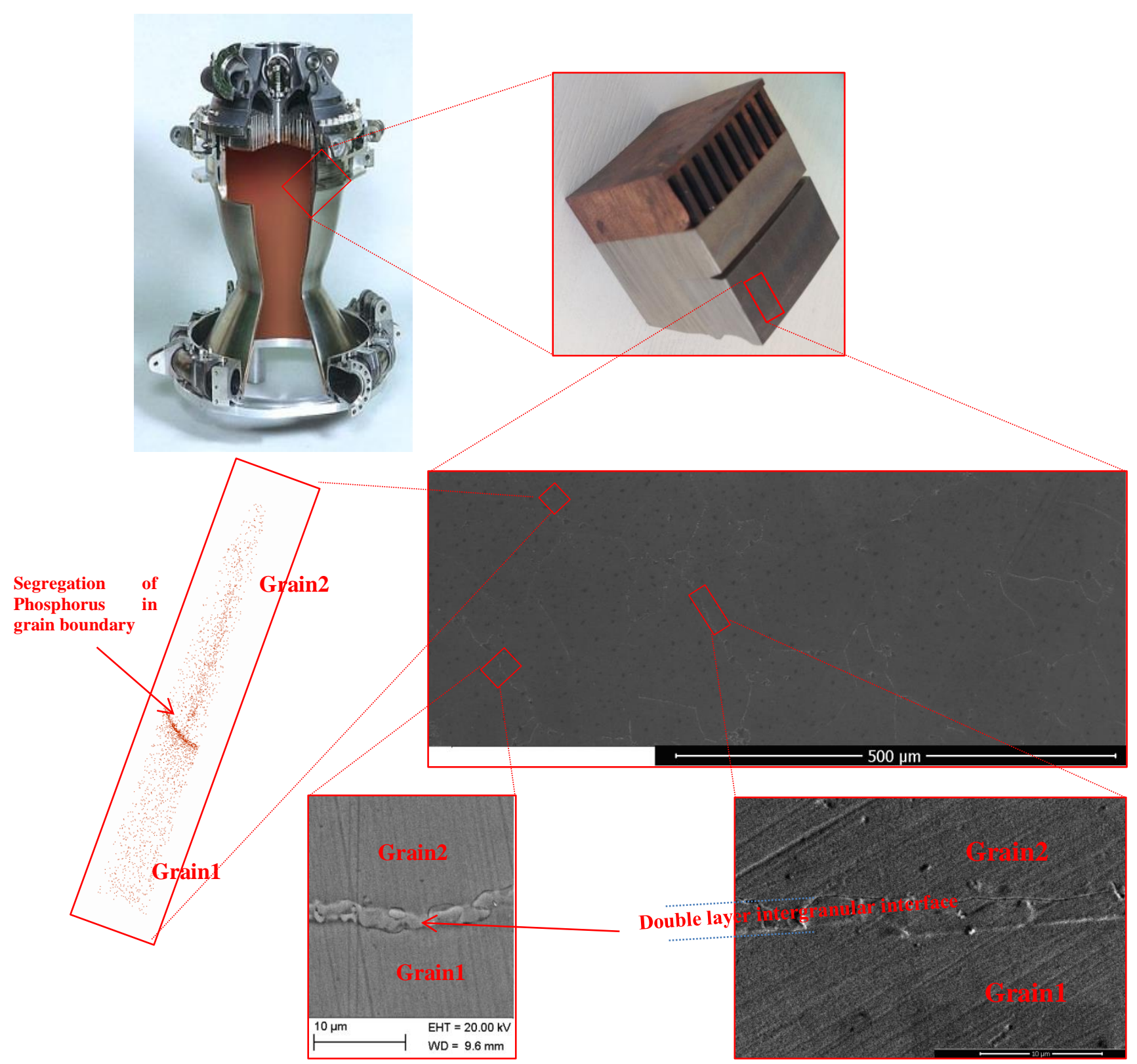

Figure 1: Intergranular double layer micro precipitates in nickel based polycrystalline superalloy used in space shuttle combustion chamber (NB precipitate hardened) and an atom probe study showing the segregation of phosphorus along a grain boundary in nickel based superalloy (Atom probe studies carried out at Chalmers University, Gothenburg, Sweden with Dr. Mattias Thuvander). 


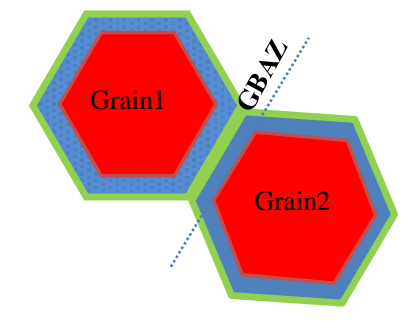

(a)

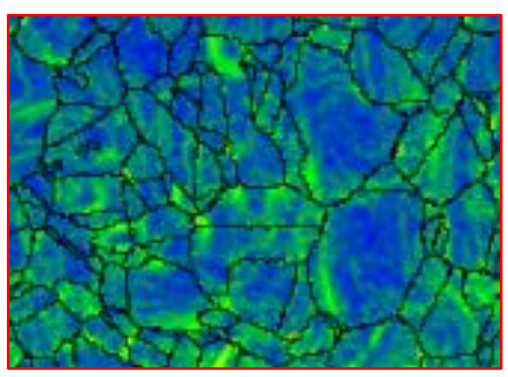

(c) EBSD image showing the local misorientation

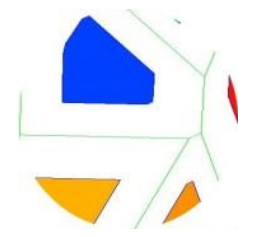

(f)

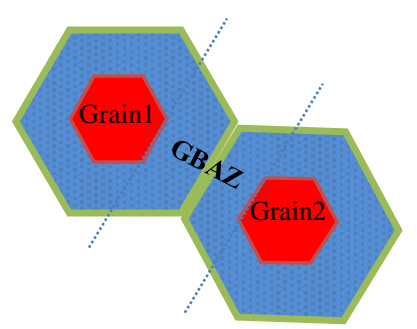

(b)
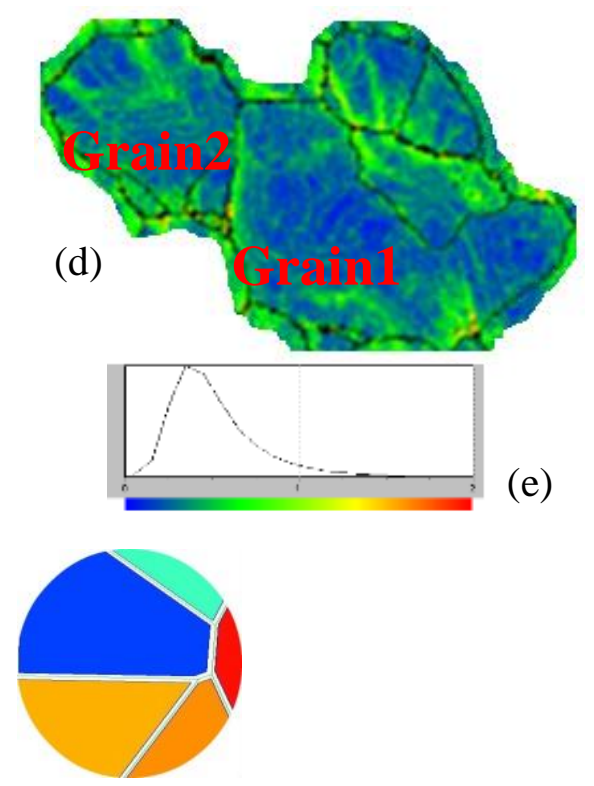

$(\mathrm{g})$

Figure 2: Schematic view of polycrystalline microstructure with double layer intergranular interface. (a) and (b) show the schematic view of polycrystalline material with double layer intergranular interfaces with different GBAZ thicknesses. (c) EBSD result showing the local misorientation in nickel microstructure, (d) shows the close view of local misorientations; (e) shows the local misorientation colour counters. (f) and (g) show the close up view of the computationally simulated multi-phase polycrystalline microstructure model with two different double layer intergranular interface thickness. 
(a) EBSD analysis results of micro polycrystalline microstructure.

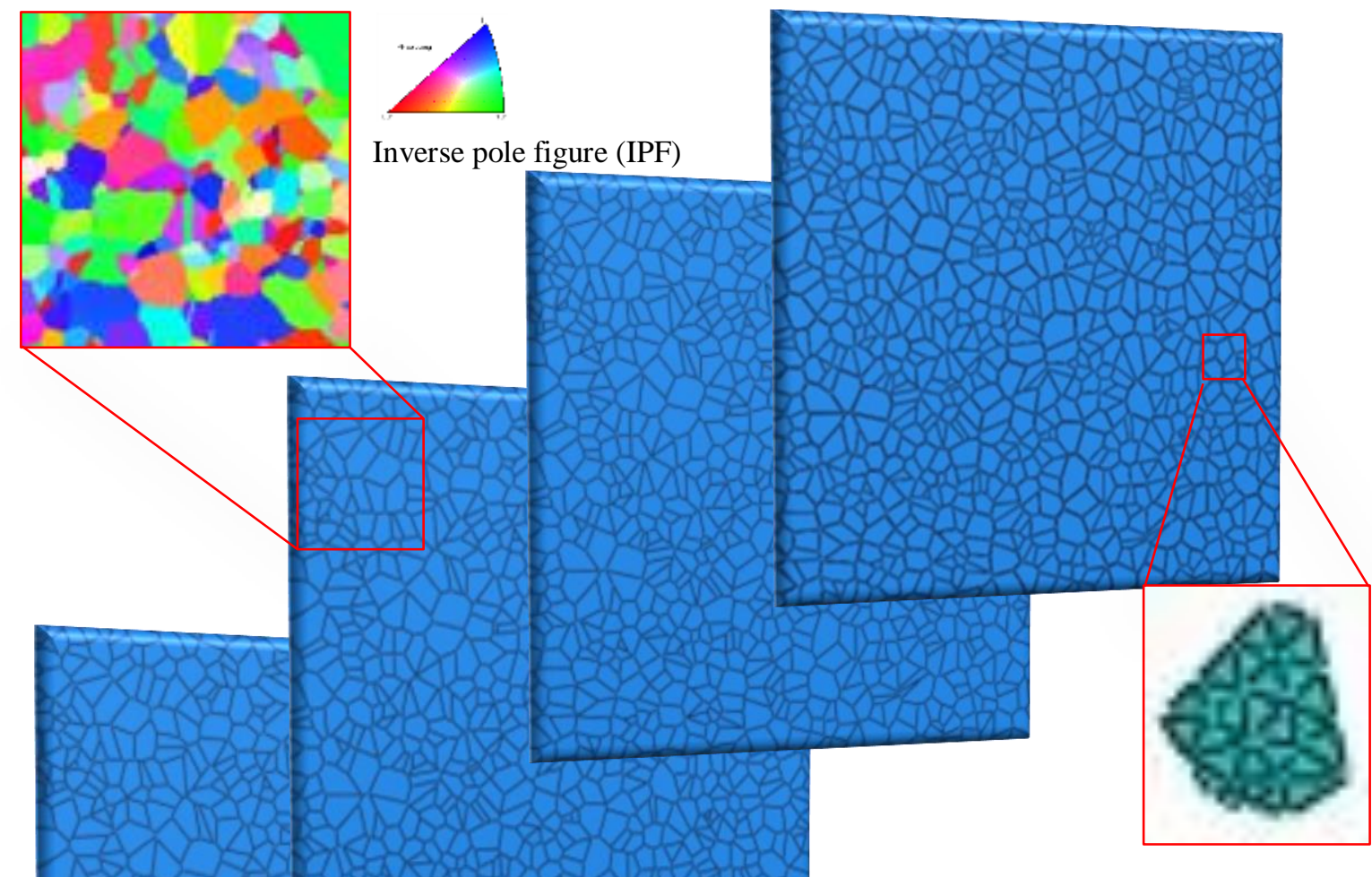

Close view of grain and its mesh

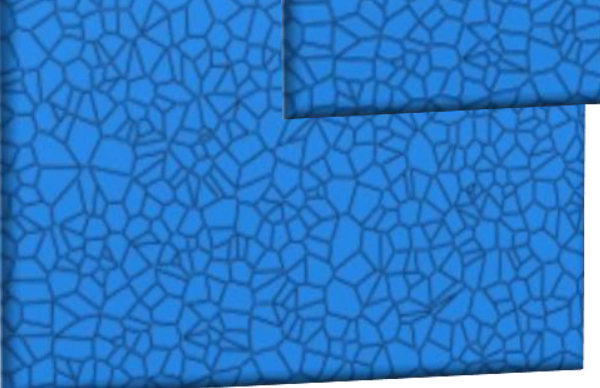

(b) Computationally simulated multi-phase polycrystalline microstructures with varying GBAZ thicknesses

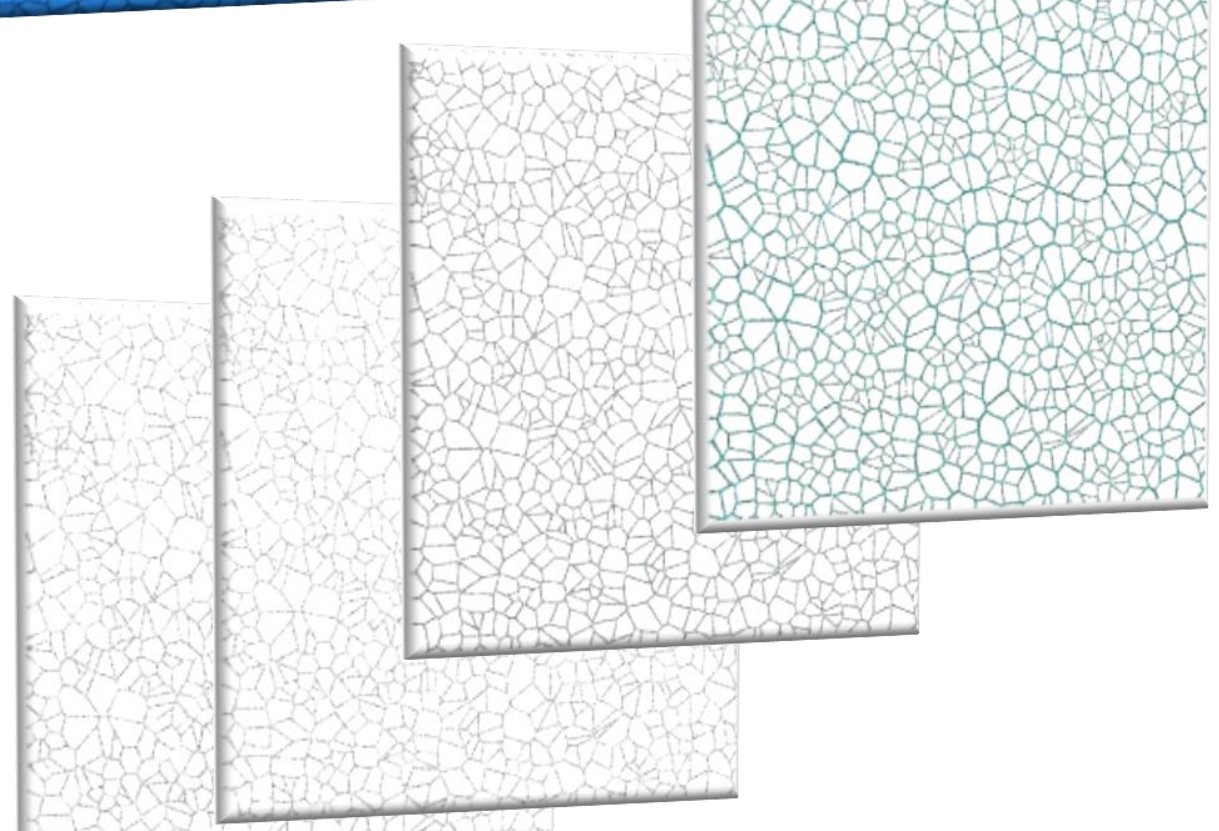

(c) Double layered intergranular interface network with different thicknesses 
Figure 3: Computationally generated multi-phase micro polycrystalline microstructures with and (a) EBSD analysis of micro polycrystalline microstructure. (b) different GBAZ and (c) double layered intergranular interface networks with different thickness

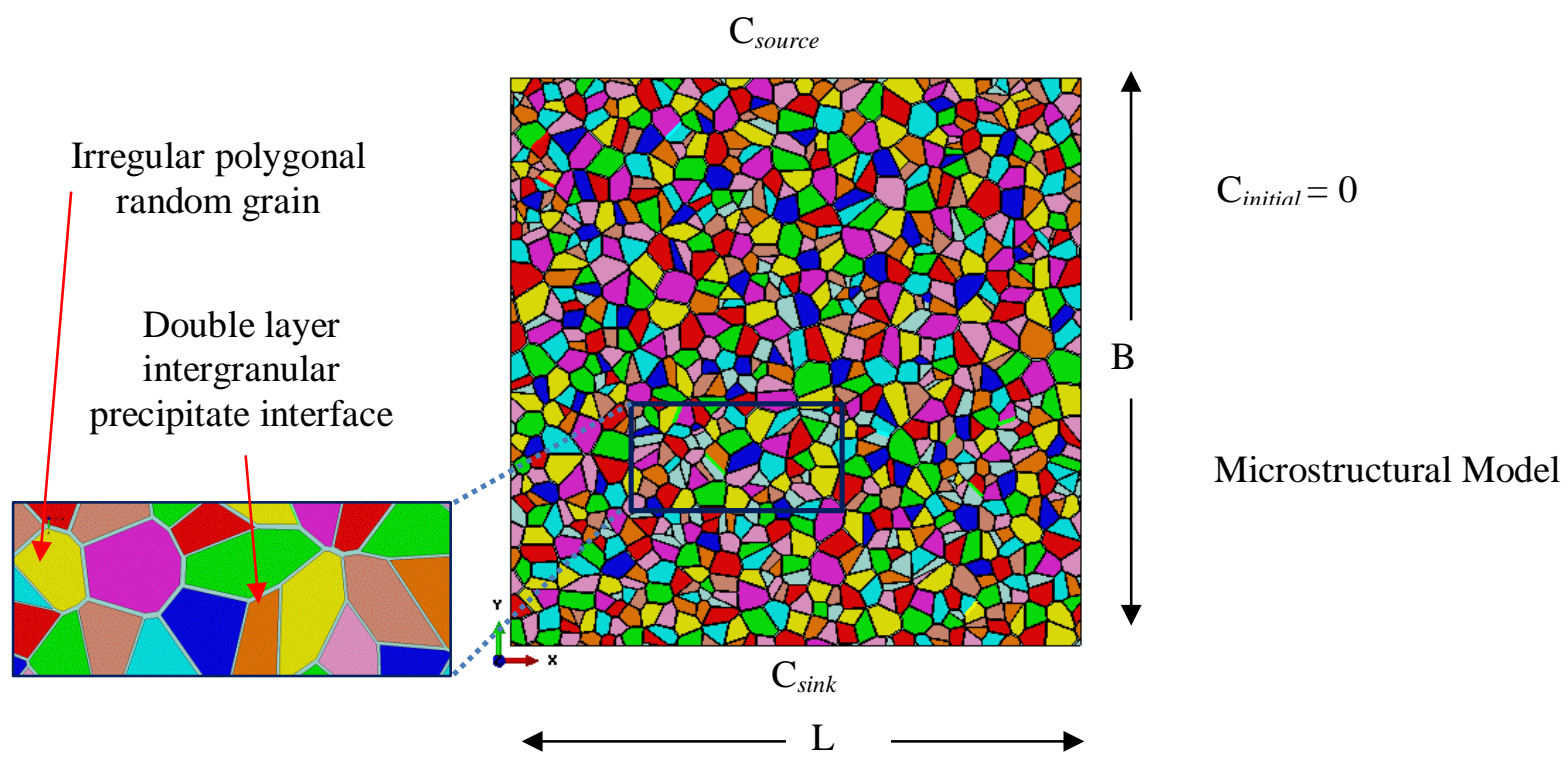

Figure 4. Microstructure and boundary conditions (hydrogen source at top face and hydrogen sink at bottom face) of the model. 


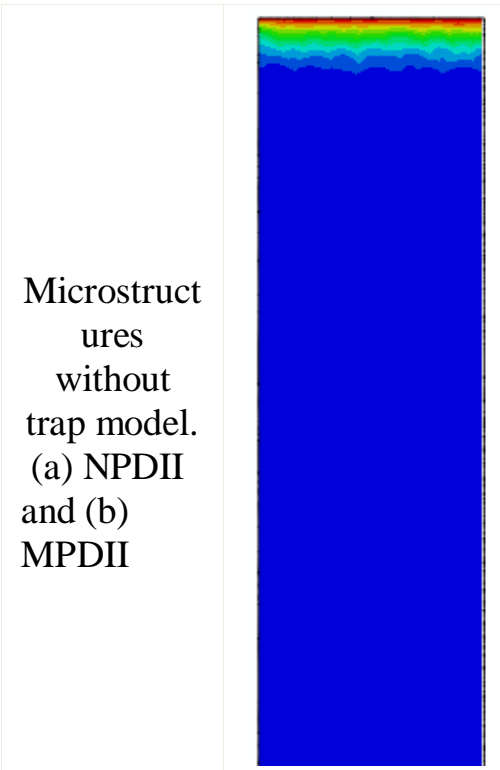

(a)

Microstruct ures with trap model.

(d) NPDII

(e) MPDII

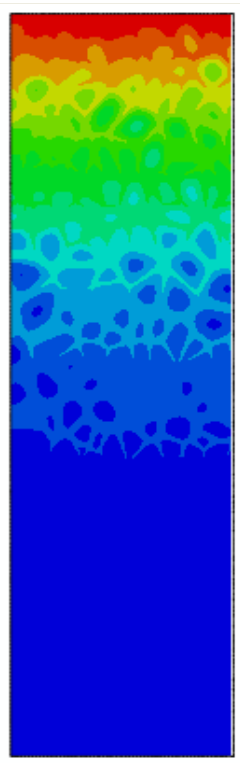

(b)

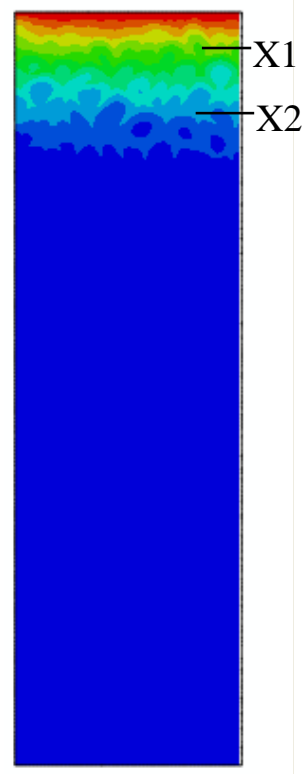

(e)

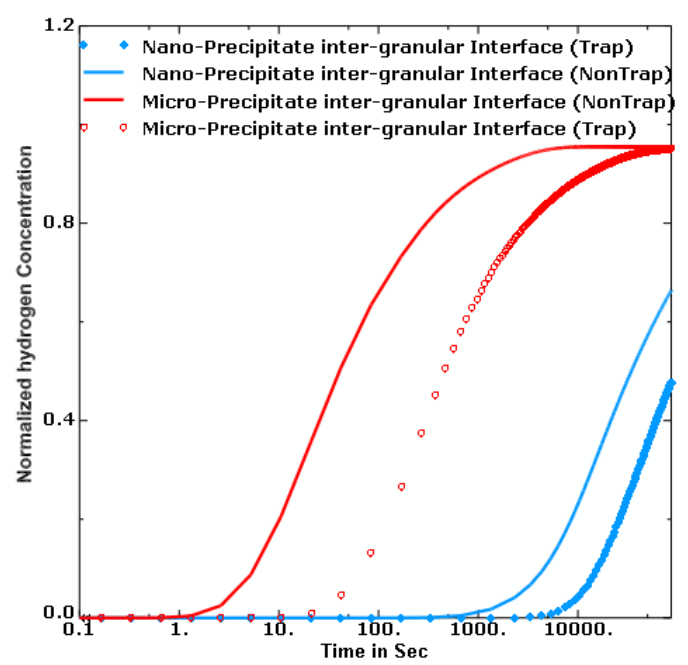

(c)
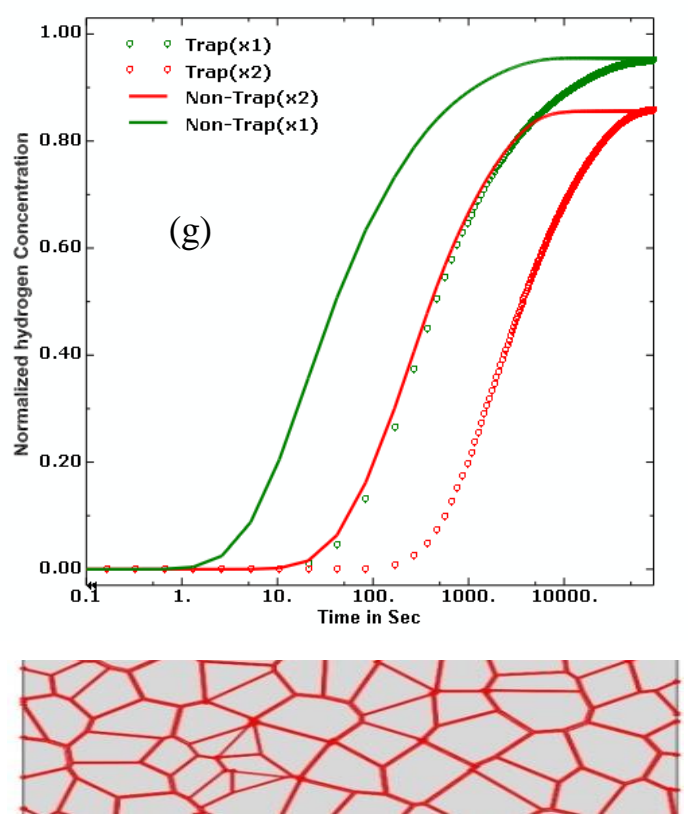

(f)

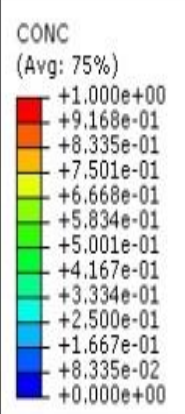

Figure 5: Hydrogen distribution and segregation with and without trapping for NPDII and MPDII interfaces in multi-phase polycrystalline materials. (NB hydrogen diffusivity in MPDII and NPDII is $4 \times 10^{-12} \mathrm{~m}^{2} \mathrm{~s}^{-1}$; Diffusivity in GI for both models is $9 \times 10^{-14} \mathrm{~m}^{2} \mathrm{~s}^{-1}$; elapsed time is $10^{4} \mathrm{~s}$ ). Colour contours are the same for all results. (c) Shows the normalized hydrogen concentration of multi-phase polycrystalline microstructure for NPDII and MPDII interfaces with and without the trap model. (f) The red color shows the precipitate double layer intergranular interface where the trap elements are implemented; (g) shows the normalized hydrogen concentration for the MPDII interface model with and without the trap model at two different positions. 
Distribution of normalized hydrogen concentration after 1 hour

(a)

(b)

(c)

(d)
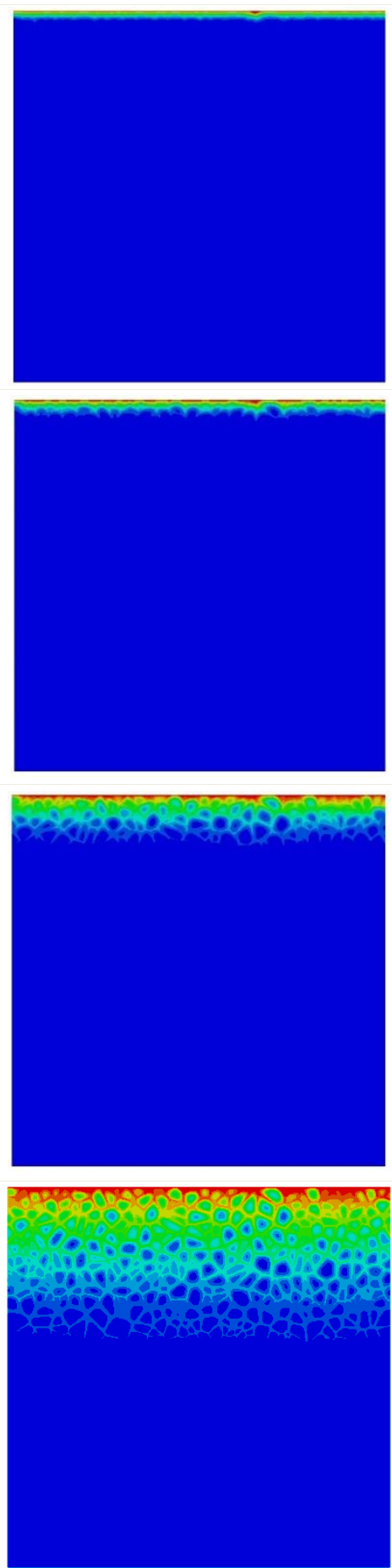

Distribution of normalized hydrogen concentration after 1day

Figure 6. Contours of hydrogen concentration in a computationally simulated polycrystalline material for increasing GBAZ thicknesses (a), (b), (c) and (d) are for GBAZ thicknesses of $0.005,0.05,0.5$ and $5 \mu \mathrm{m}$ respectively, after 1 hour (middle) and 1 day (right). Note: GI and GBAZ diffusivities used are $9 \times 10^{-14}$ and $4 \times 10^{-12} \mathrm{~m}^{2} \mathrm{~s}^{-1}$ respectively used in all simulations.
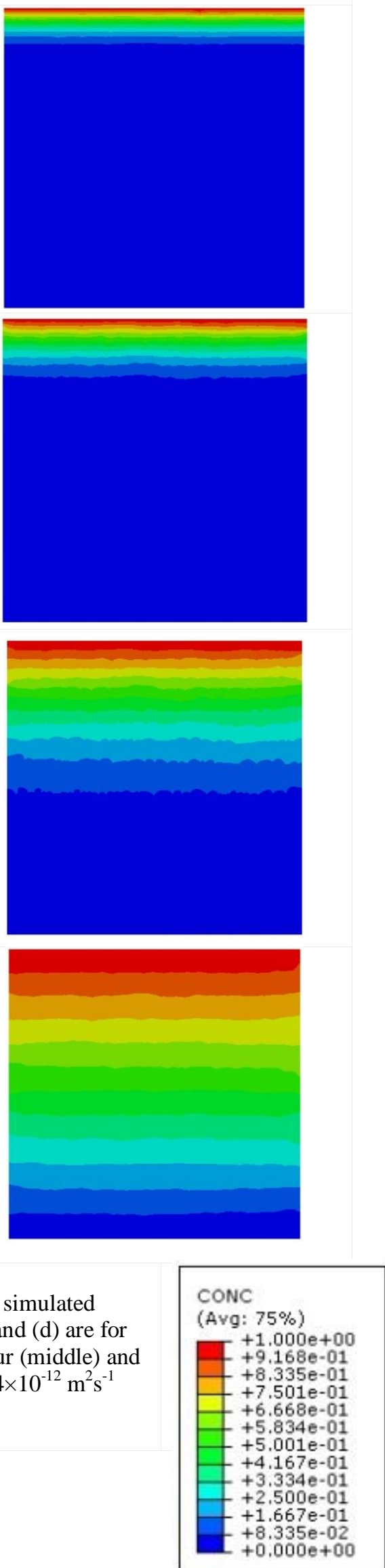


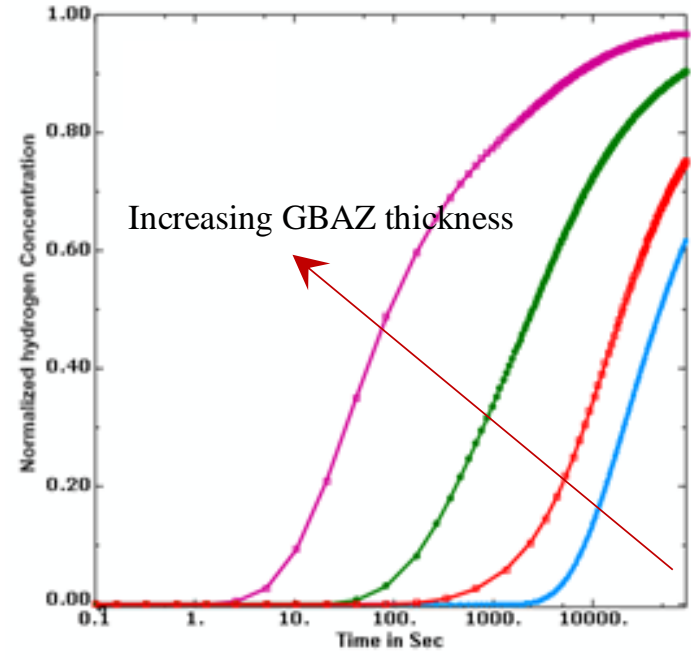

(a)

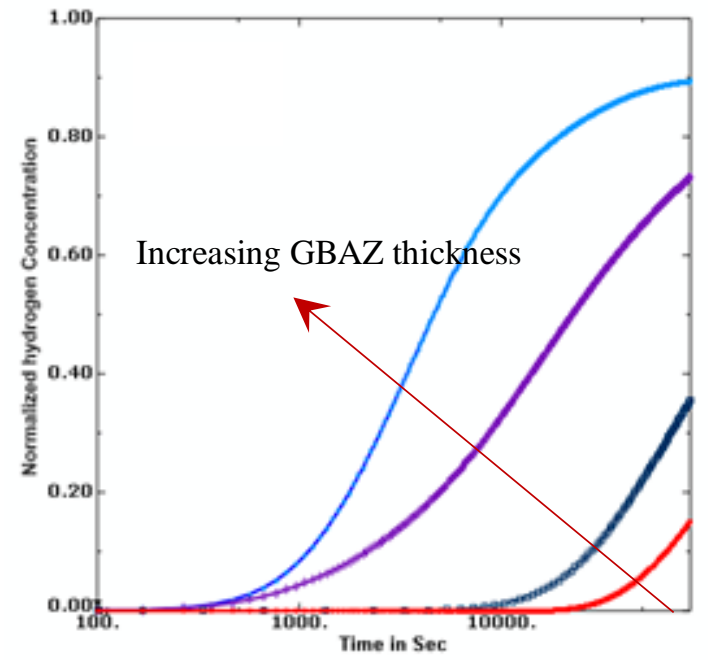

(b)

Figure 7. Normalized hydrogen concentration of polycrystalline microstructures with four different GBAZ thicknesses, $0.005,0.05,0.5$ and $5 \mu \mathrm{m}$, at two different positions. (a) $70 \mu \mathrm{m}$ and (b) $200 \mu \mathrm{m}$ from the top surface of the computational grid.

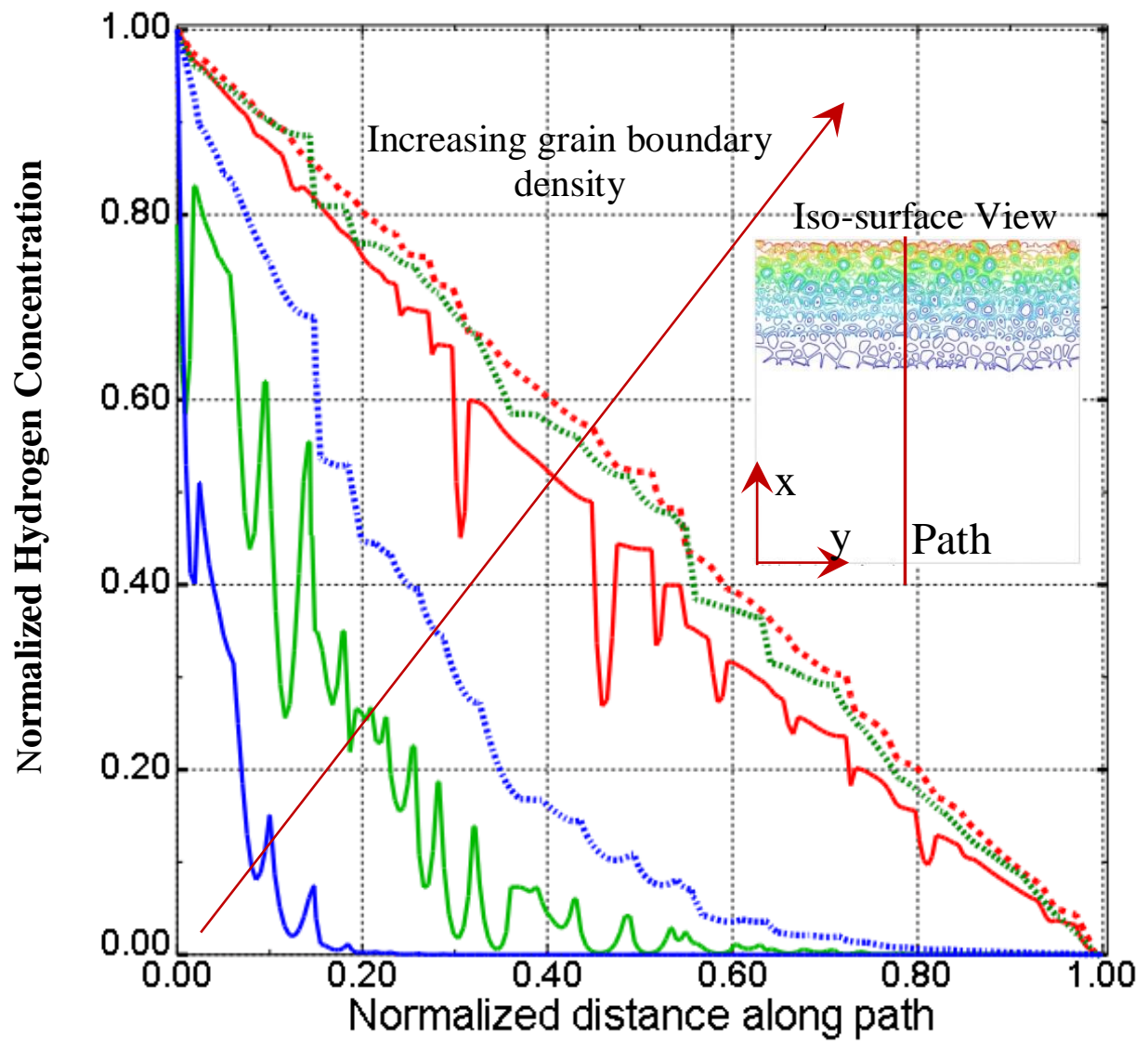

Figure 8. Normalized hydrogen concentration along the vertical path shown above in the polycrystalline material for GBAZ thicknesses of 0.05 (blue), 0.5 (green) and 5 (red) $\mu \mathrm{m}$ after (i) one hour (solid lines) and (b) one day (dotted lines). 


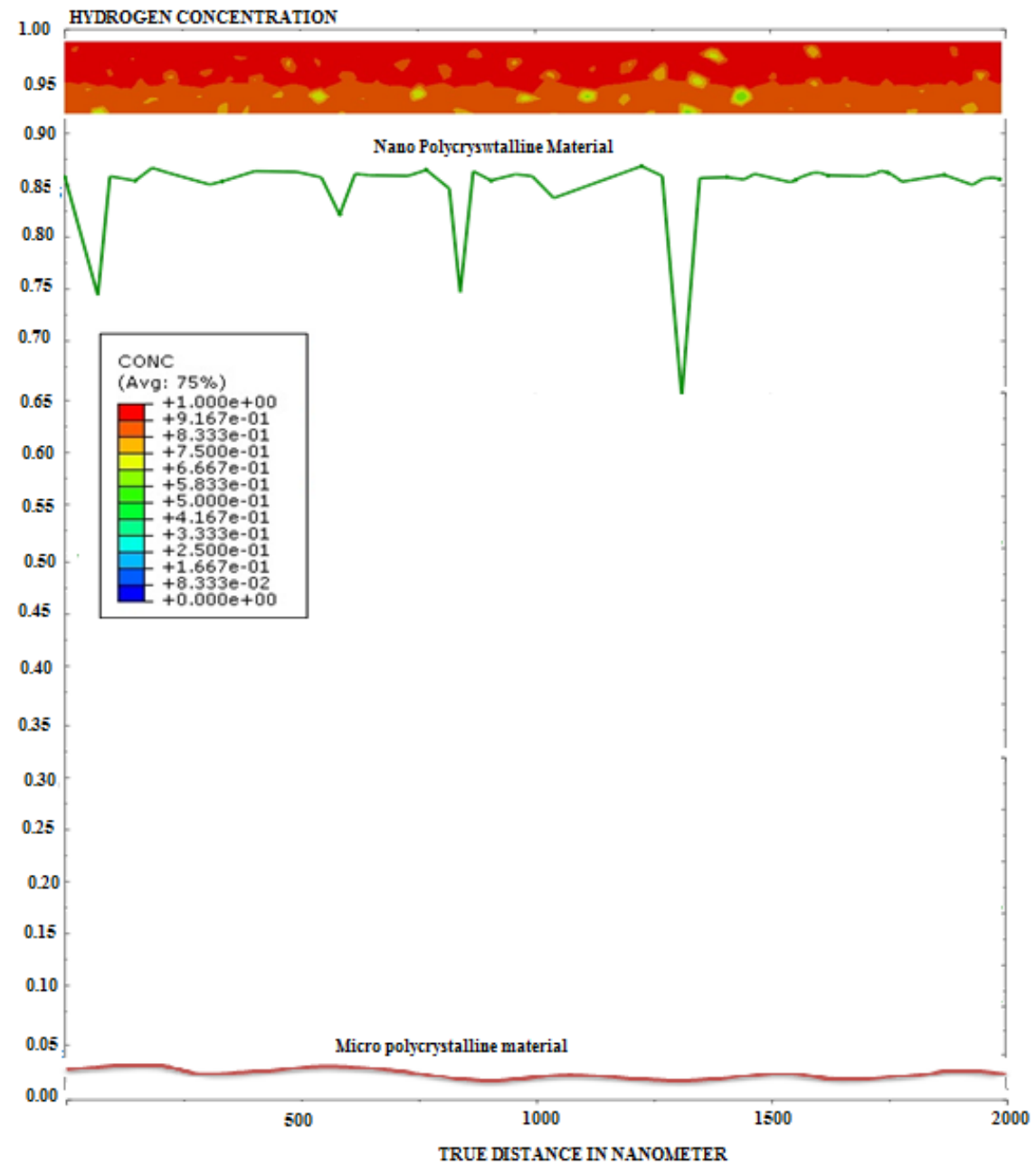

(a)

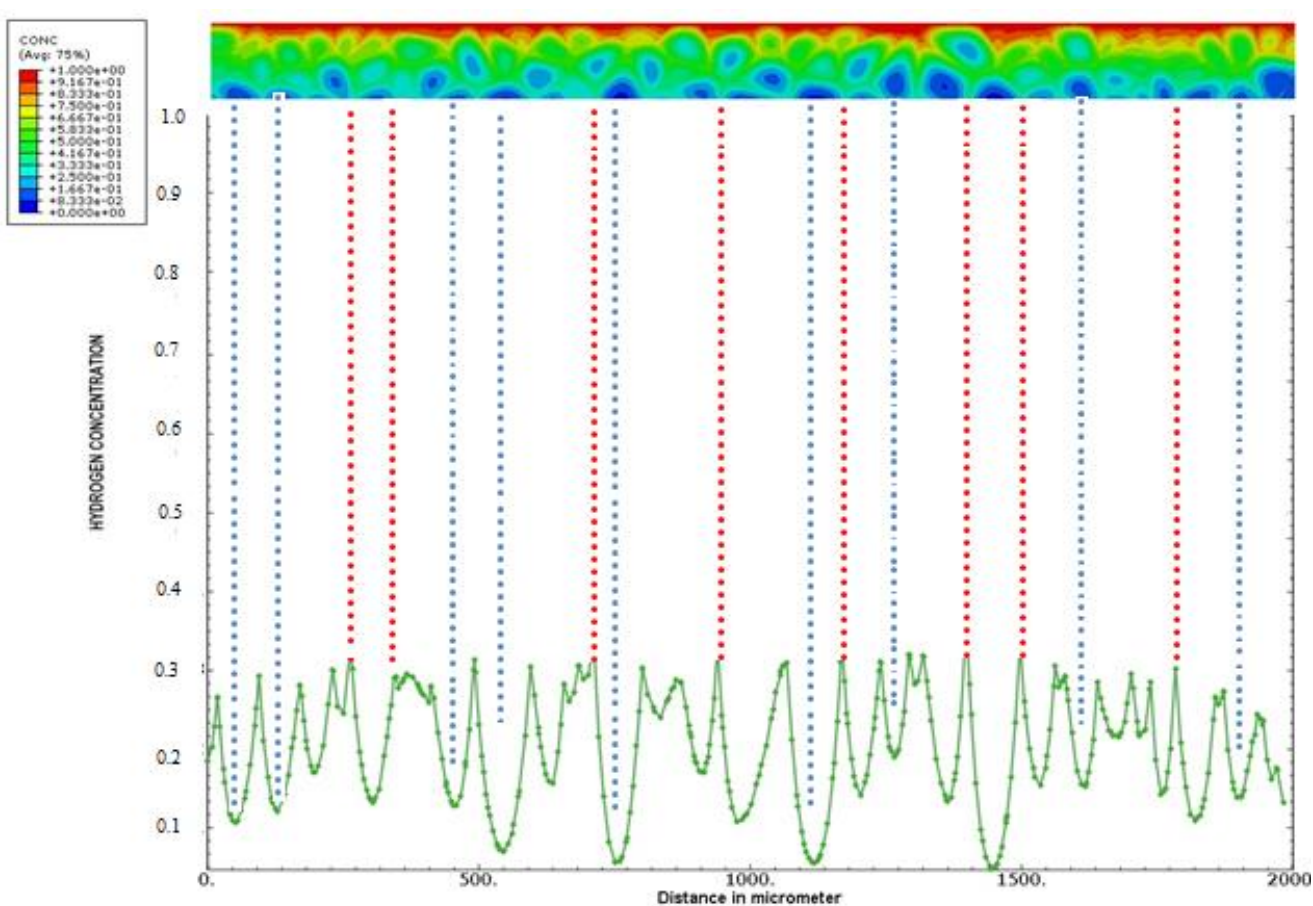

(b) 


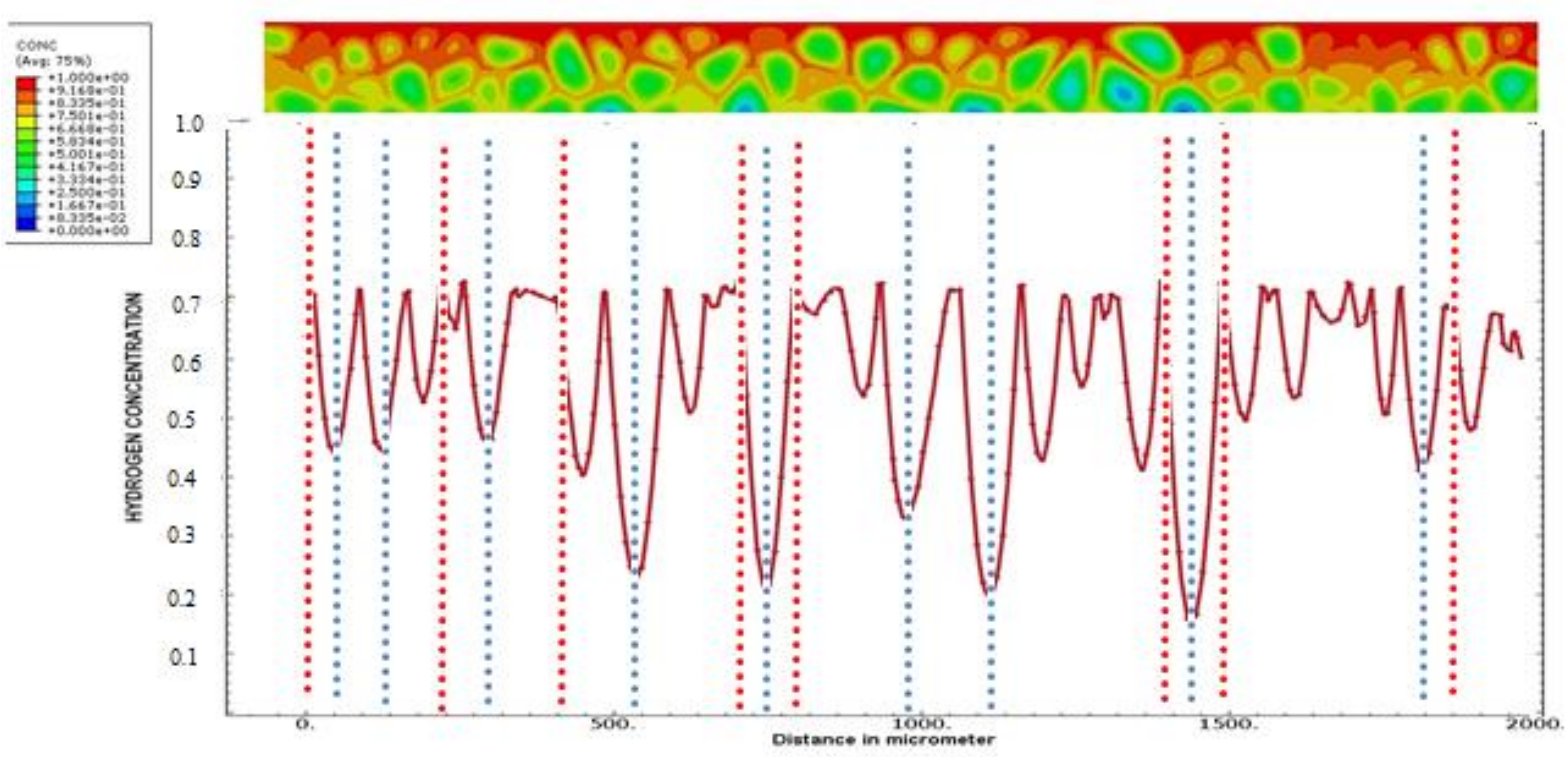

(c)

Figure 9. Segregations/Accumulation of hydrogen along intergranular grain boundaries and inhomogeneous hydrogen diffusion processes along grain boundaries and within grains for (a) nano polycrystalline material with nano scale grains after one second and micro polycrystalline material after one second, (b) and (c) micro polycrystalline material with two different GBAZ thicknesses after 1 hour. Note: red dotted lines show the Segregations/accumulation of hydrogen in grain boundaries and blue dotted lines show the hydrogen concentration in grains). (Note: GBAZ diffusivity $=4 \times 10^{-12} \mathrm{~m}^{2} \mathrm{~s}^{-1}$ and GI diffusivity $=9 \times 10^{-14} \mathrm{~m}^{2} \mathrm{~s}^{-1}$ ). Note: Colour contours are same for all results). 


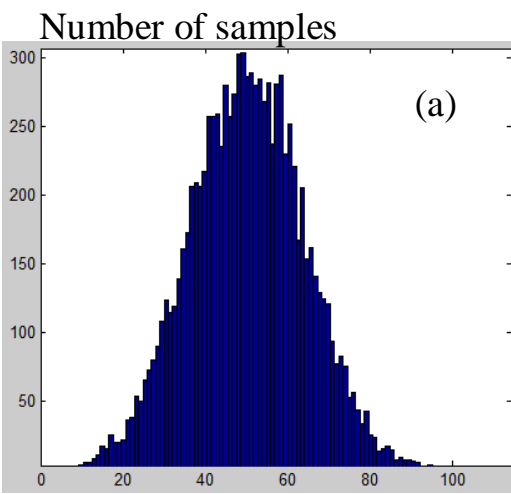

Segregation factor (S)

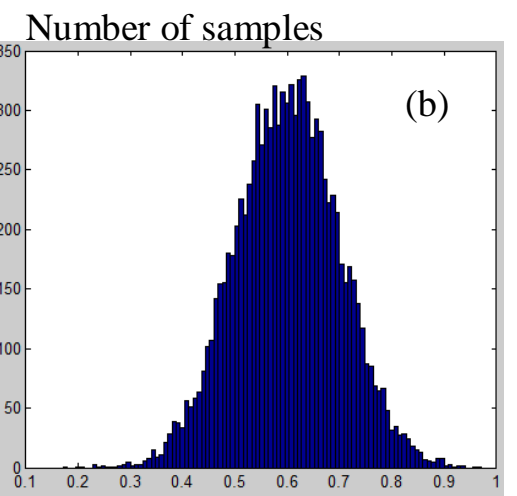

$\mathrm{C}_{\mathrm{L}}(\mathrm{ppm})$

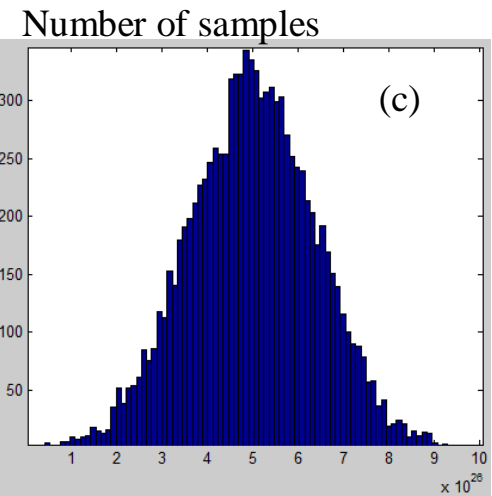

$N_{t}^{G N D}\left(\mathrm{~m}^{-3}\right)$

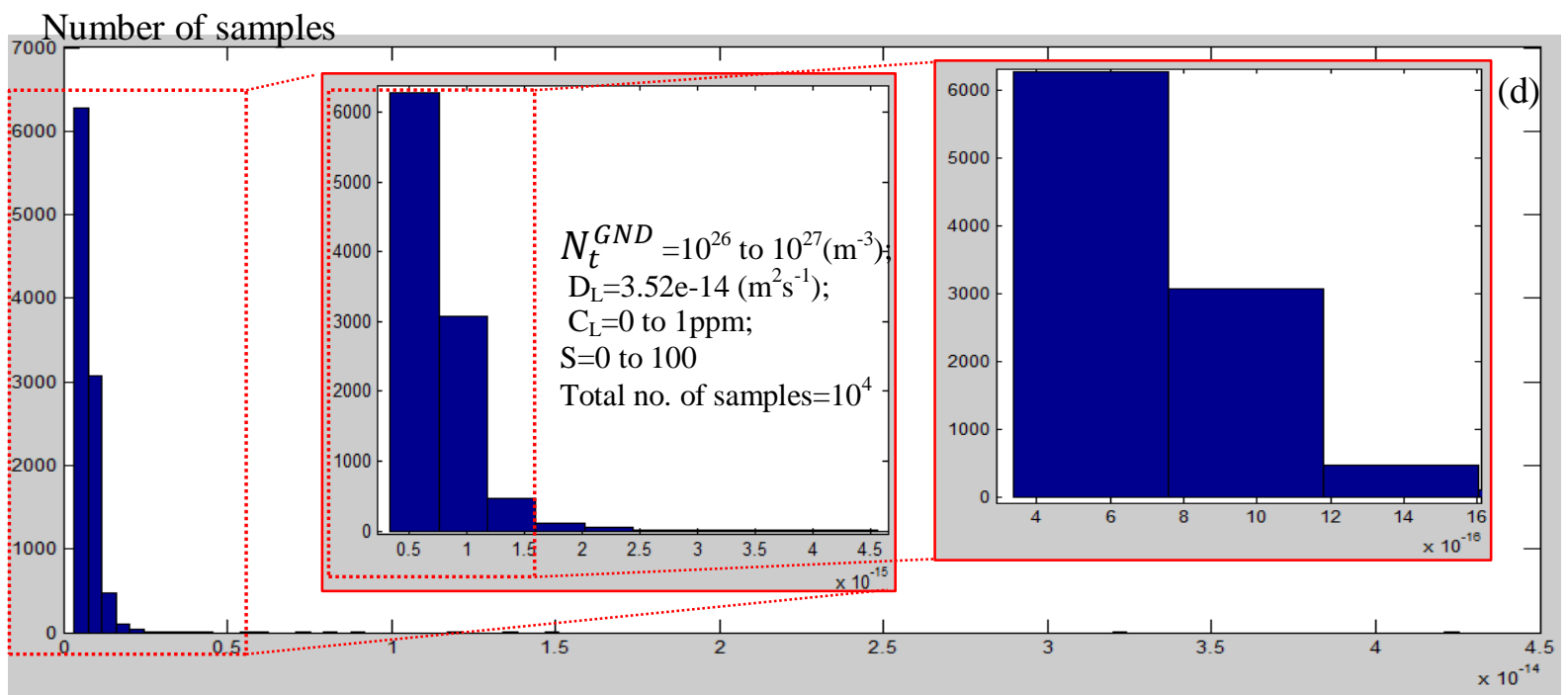

$$
D_{\text {eff }}\left(m^{2} s^{-1}\right)
$$
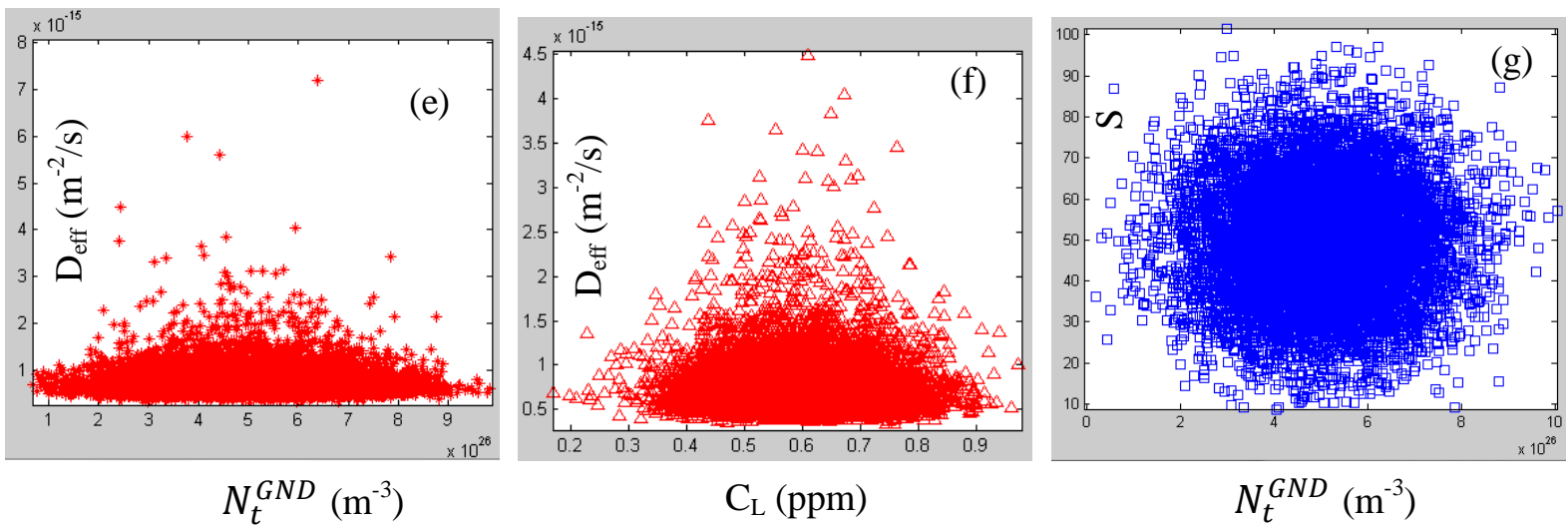


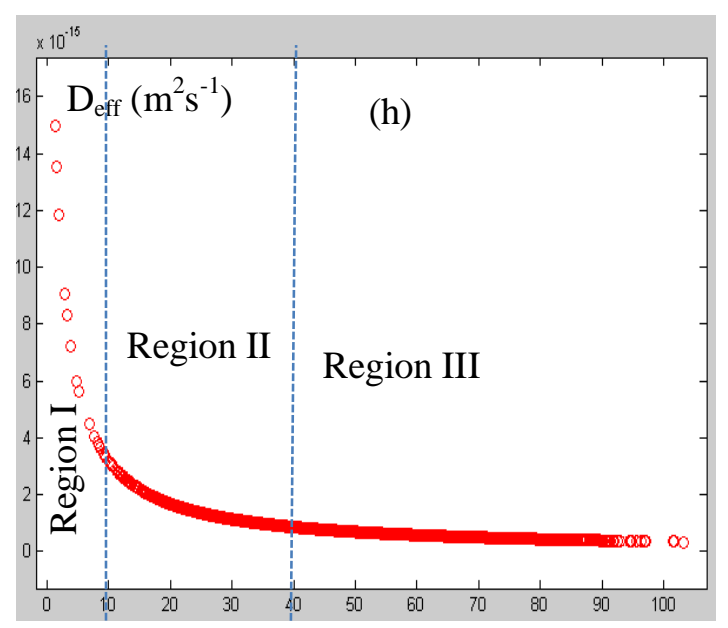

Segregation factor (S)

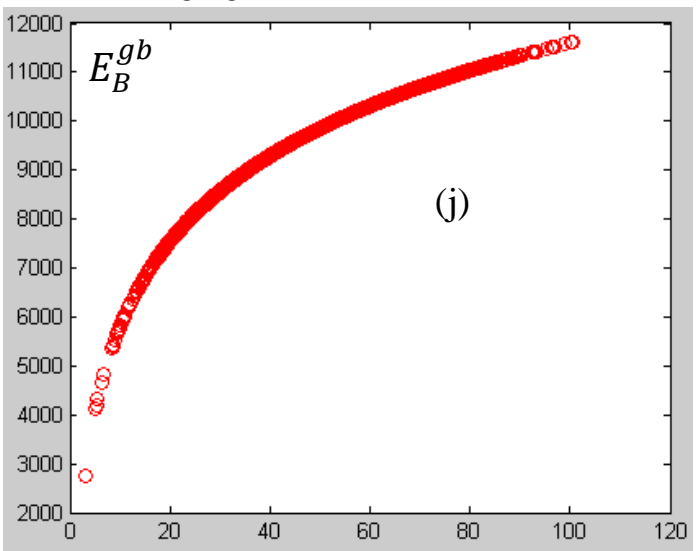

Segregation factor (S)

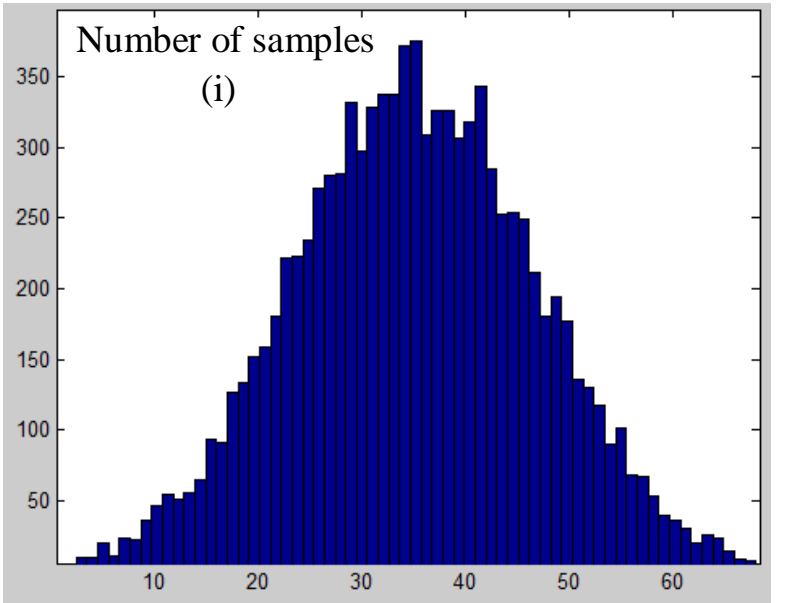

GB Misorientation $\left(\theta^{\circ}\right)$

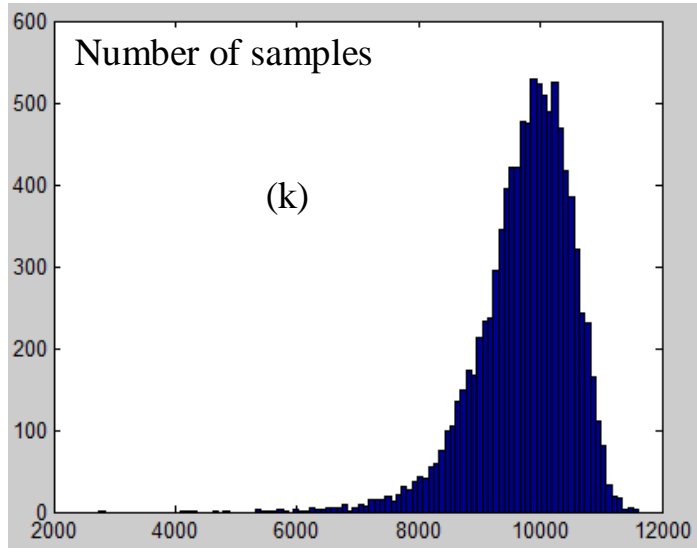

Average grain boundary trap site binding energy $\left(E_{B}^{g b}\right)$

Figure 10. Monte Carlo simulation results of effective diffusivity $\left(\mathrm{D}_{\text {eff }}\right)$ for 10000 sample distributions assuming a lattice concentration $\left(\mathrm{C}_{\mathrm{L}}\right)$ from 0 to $1 \mathrm{ppm}$, density of trapping sites based on grain boundary misorientation $\left(\mathbf{N}_{\mathbf{t}}^{\mathbf{G N D}}\right.$ ) from $10^{26}$ to $10^{27}\left(\mathrm{~m}^{-3}\right)$, segregation factors (S) from 0 to 100 and a lattice diffusivity of $3.52 \times 10^{-14} \mathrm{~m}^{2} \mathrm{~s}^{-1}$. (a) distribution of segregation factors, (b) distribution of lattice concentration of hydrogen, (c) density of trapping sites based on grain boundary misorientation, (d) effective diffusion of hydrogen in polycrystalline material, its closed view and data used for statistical samples floatation's, (e) effective diffusivity of hydrogen as a function of density of trapping sites, (f) effective diffusivity of hydrogen as a function of lattice hydrogen concentration, ( $\mathrm{g}$ ) segregation factor distribution as a function of density of trapping sites, (h) effective diffusivity as a function of segregation factors, (i) statistical distribution of GB misorientations, (j) segregation factors versus average grain boundary trapping sites binding energy (increasing segregation factor with increasing $\mathbf{E}_{\mathbf{B}}^{\mathbf{g b}}$ ) and (k) Shows the distribution of average grain boundary trapping sites binding energy. 

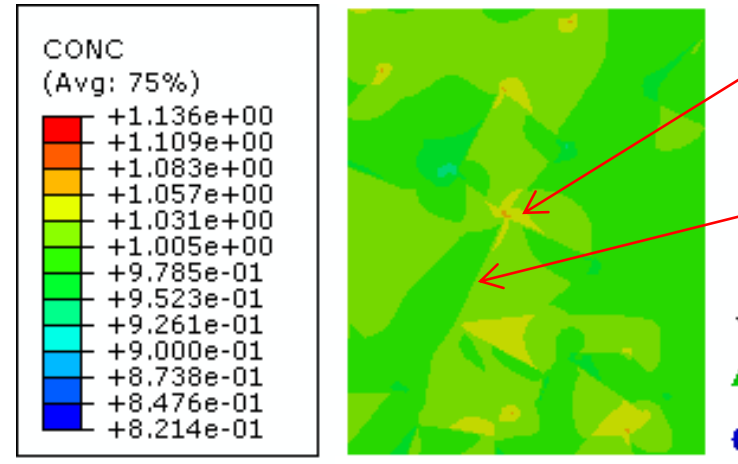

(a)
$\mathrm{S}\left(\theta=30^{\circ}\right)=1.263$ (At Triple Junction)

$\mathrm{S}\left(\theta=30^{\circ}\right)=1.001$ (at GB)

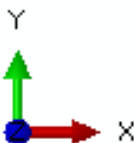
$\mathrm{S}=0.7$ (at GB)

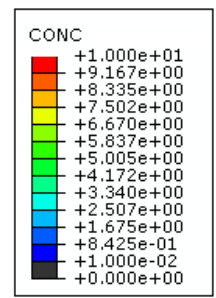

(b)

$$
\mathrm{S}=0.3
$$
(at GB)

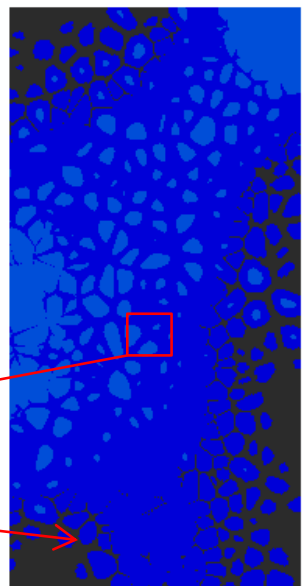

Figure 11 shows the hydrogen segregations results of the Monte Carlo simulation coupled with FE multiscale multiphase continuum trap model based on (a) GB misorientations and (b) without GB misorientation and its segregations factors $(\mathrm{S})$. 